\title{
Quantum entanglement theory in the presence of superselection rules
}

\author{
Norbert Schuch, Frank Verstraete, and J. Ignacio Cirac \\ Max-Planck-Institut für Quantenoptik, Hans-Kopfermann-Str. 1, D-85748 Garching, Germany.
}

\begin{abstract}
Superselection rules severly constrain the operations which can be implemented on a distributed quantum system. While the restriction to local operations and classical communication gives rise to entanglement as a nonlocal resource, particle number conservation additionally confines the possible operations and should give rise to a new resource. In [Phys. Rev. Lett. 92, 087904 (2004), quant-ph/0310124] we showed that this resource can be quantified by a single additional number, the superselection induced variance $(\mathrm{SiV})$ without changing the concept of entanglement. In this paper, we give the results on pure states in greater detail; additionally, we provide a discussion of mixed state nonlocality with superselection rules where we consider both formation and distillation. Finally, we demonstrate that $\mathrm{SiV}$ is indeed a resource, i.e., that it captures how well a state can be used to overcome the restrictions imposed by the superselection rule.
\end{abstract}

PACS numbers: 03.67.-a,03.65.Ud,03.67.Mn

\section{INTRODUCTION}

One of the most interesting results in quantum information theory has been the discovery that the amount of nonlocality contained in a bipartite quantum system can be quantified by a single number, the entropy of entanglement (EoE). Asymptotically, multiple copies of any two states can be converted into each other and thus into singlets provided that the total EoE is conserved [1]. On the other hand, entanglement is the key resource for some of the most interesting tasks in quantum information, as teleportation [2] and dense coding 3].

Entanglement has its origin in the restriction to those transformations which can be implemented by local operations and classical communication (LOCC) [4, 5]. In the same way, any additional restriction should lead to another nonlocal quantity and thus to new effects and applications. It has been noted by Popescu [6] that in many physical systems of interest such a restriction is given by a superselection rule (SSR). In this work, we will consider particle number conservation as a superselection rule; this is motivated, e.g., by recent quantum optical experiments on cold atomic gases. Indeed, the notion of entanglement is affected by the additional restrictions [7, 8], and new protocols arise, e.g., perfect data hiding [9] becomes possible 7]. On the other hand, it has been shown [7, 10] that the extra resource of a shared reference frame (i.e., a nonlocal state) allows to overcome the restrictions imposed by the SSR (note that [10] also adressed non-Abelian SSR); conversely, private reference frames restrict the possible operations of an eavesdropper and can thus be employed for cryptographic tasks 11].

In [12], we have shown that the nonlocality contained in a bipartite state subject to SSR can be quantified by only one additional number, the superselection induced variance (SiV): any two states can be interconverted asymptotically as long as the total EoE and SiV are conserved. In this paper, we prove this result in greater detail and extend it to mixed states. We start by discussing how the majorization criterion [13] which governs the conversion of quantum states has to be changed when SSR are present, and show that it asymptotically converges to the conservation of EoE (as it is the case without SSR) and SiV. We give a detailed proof of this result for arbitrary states and show that it motivates the definition of two different types of standard forms for $\mathrm{SiV}$ which carry a linear resp. logarithmic amount of EoE.

While there exist pure states which carry only EoE, there are no pure states which contain solely SiV. On the other hand, it has been demonstrated [7] that there exist separable but nonlocal mixed states, i.e., states which have a separable decomposition and thus do not contain EoE, but are still nonlocal as all these decompositions violate the SSR and therefore should contain $\mathrm{SiV}$. In order to make these statements quantitative, we extend the concepts of EoE and SiV to mixed states subject to SSR. One natural way to do this is to consider the amount of pure states resources needed to create the state [14]; we show that this extension can be done in a meaningful way and that there indeed exist states which contain $\mathrm{SiV}$ but no EoE. The converse way is to ask whether it is possible to distill pure state resources from some mixed state 15]; we provide ways to distill both EoE and SiV, and we show that it is even possible to distill the SiV contained in separable states.

EoE is a resource - it allows to overcome the LOCC restrictions by teleportation. It is reasonable to assume that any restriction leads to a nonlocal quantity which in turn allows to overcome this restriction. Indeed, we give evidence that $\mathrm{SiV}$ can be used as a resource which allows to overcome the additional restrictions imposed by the SSR in a bipartite setting (cf. [7, 12]). Therefore, we will use two tasks: distinguishing locally undistinguishable quantum states and teleporting states with nonconstant local particle number [7]. We will show that not only pure states can be used as share reference frames for these tasks, but that there even exist separable states which together with one ebit of entanglement allow to perfectly teleport one qubit and thus to overcome all restrictions. Still, we find that there is a fundamental difference between EoE and $\mathrm{SiV}$ as a resource, as a finite amount of nonlocality does not allow to perfectly overcome the re- 
strictions which is due to the structure of the underlying Hilbert space [12].

The paper is organized as follows. In Sec. III we introduce the concept of a superselection rule and show how it restricts the operations which can be implemented in a bipartite setting. In Sec. IIII we consider the conversion of pure states. We start with the conversion of single copies, which motivates the definition of $\mathrm{SiV}$ as a nonlocal monotone; then, we prove that asymptotically all states can be converted given that both $\mathrm{SiV}$ and $\mathrm{EoE}$ are conserved. Sec. IV] is devoted to mixed state nonlocality. First, we discuss formation of mixed states; beyond other results, we provide explicit formulas for the case of qubits. Second, we give different methods for the distillation of both EoE and SiV independently as well as simultaneously. Finally, Sec. $\mathrm{V}$ discusses $\mathrm{SiV}$ as a resource; there, we quantify how well states with SiV can be used as shared reference frames which allow to overcome the new restrictions, and we demonstrate that one ebit of entanglement is still sufficient for teleportation.

\section{PARTICLE NUMBER CONSERVATION AS A SUPERSELECTION RULE}

In this paper, we focus on particle number conservation as a SSR, but the results also apply to charge and other discrete quantities. In this case, the Hilbert space of the system $\mathcal{H}$ can be decomposed into a direct sum $\mathcal{H}=\bigoplus_{N=0}^{\infty} \mathcal{H}_{N}$ of the eigenspaces of the particle number operator $\hat{N}$, and the SSR imposes that for any operator $\mathcal{O},[\mathcal{O}, \hat{N}]=0$ must hold; thus, any operator can be written as a sum of operators $\mathcal{O}_{N}$ which have support on $\mathcal{H}_{N}$ only, $\mathcal{O}=\bigoplus_{N=0}^{\infty} \mathcal{O}_{N}$, and thus

$$
\mathcal{O}=\sum_{N} P_{N} \mathcal{O} P_{N}
$$

where $P_{N}$ projects onto $\mathcal{H}_{N}$. As the same restriction holds for the admissible density operators, all states can be converted into each other, and no interesting new effects can be found.

Therefore, we consider SSR in a bipartite setting. Then, we have local particle number operators $\hat{N}_{A}$ and $\hat{N}_{B}$, and the total particle number operator is given by

$$
\hat{N}_{A B}=\hat{N}_{A} \otimes \mathbb{1}_{B}+\mathbb{1}_{A} \otimes \hat{N}_{B} .
$$

While the admissible states have to commute with the global particle number operator $\hat{N}_{A B}$, the local operations have to commute with the local particle number operators $\hat{N}_{A}$ and $\hat{N}_{B}$. This restriction is stronger than the one given by the bipartite setting alone and should therefore lead to a new nonlocal resource. More precisely, the operations on subspaces with fixed total particle number $N=N_{A B}$ are given by

$$
\mathcal{O}_{N}^{A B}=\bigoplus_{N_{A}+N_{B}=N}\left(\mathcal{O}_{N_{A}}^{A} \otimes \mathbb{1}_{N_{B}}^{B}\right)
$$

(and vice versa) - in addition to the restriction to products $\mathcal{O}^{A} \otimes \mathbb{1}$ imposed by the bipartite setting, a direct sum structure arises from the SSR. This product vs. sum structure will reappear throughout the paper and is the reason for some fundamental differences between EoE (arising for the product structure) and $\mathrm{SiV}$ (arising from the direct sum).

The restriction to block-diagonal operations, Eq. (1), can be relaxed by adding ancilla modes with $m_{0}$ particles, performing a block-diagonal unitary $U$, and measuring resp. tracing out the ancillas. Then, the admissible (POVM/Kraus) operators are given by $\mathcal{O}=P_{m}^{\text {anc }} U P_{m_{0}}^{\text {anc }}$; by applying (11) to $U$, this leads to $\mathcal{O}=\sum_{N} P_{N+\Delta} \mathcal{O} P_{N}$ (resp. $[\hat{N}, \mathcal{O}]=\Delta \mathcal{O}, \Delta$ might differ for each $\mathcal{O}$ ): $\mathcal{O}$ can shift the particle number by some $\Delta$. (Note that $\mathcal{O}^{\dagger} \mathcal{O}$ remains block-diagonal). As most results of this paper are only affected marginally by including ancillas, we will usually neglect them and just briefly comment on their effect as appropriate.

At the end of this section, let us introduce a few notational conventions. Logarithms are taken to the basis 2 . A ket $|N\rangle$ denotes a state with $N$ particles. We will use this notation even if the underlying eigenspace is degenerate, unless the nonlocal properties under consideration depend on this degeneracy.

The restrictions imposed by the SSR on the allowed operations can be easily overcome by defining a new computational basis $|\hat{0}\rangle \equiv|01\rangle,|\hat{1}\rangle \equiv|10\rangle$ in which all states have the same particle number [7]. This motivates the definition of two different types of maximally entangled two-qubit states,

$$
|\mathrm{V}-\mathrm{EPR}\rangle=|0\rangle_{A}|1\rangle_{B}+|1\rangle_{A}|0\rangle_{B}
$$

(a "variance-EPR", as there is some variance in the local particle number), and

$|\mathrm{E}-\mathrm{EPR}\rangle=|01\rangle_{A}|10\rangle_{B}+|10\rangle_{A}|01\rangle_{B} \equiv|\hat{0}\rangle_{A}|\hat{1}\rangle_{B}+|\hat{1}\rangle_{A}|\hat{0}\rangle_{B}$

(an "entanglement-EPR", which is defined within an unrestricted subspace and only carries entanglement). The very difference between these two states will be a central issue of the paper.

\section{CHARACTERIZATION OF PURE STATES}

In this section, we characterize pure states in a bipartite setting, i.e., we determine the possible conversions by LOCC and thus quantify the nonlocality contained in a bipartite state. Without superselection rules, the majorization criterion determines whether the conversion between two bipartite pure states is possible 13,16 . The conversion of multiple copies is governed by a much simpler criterion: it has been shown [1] that multiple copies of any two states can be interconverted reversibly. The conversion ratio is determined by only one quantity which fully characterizes the nonlocal properties of a bipartite state, the entropy of entanglement (EoE). 
As we have seen in the preceding section, in addition to the tensor product structure induced by the bipartite setting the operators have to obey a direct sum structure. In this section, we show that these two structures lead to two complementary resources: while the tensor product again induces the majorization criterion and (asymptotically) EoE as a nonlocal resource, the direct sum gives rise to additional restrictions on the conversions of states and in turn leads to an own nonlocal resource.

\section{A. The single copy case}

Let us consider the following problem: given pure bipartite states $\phi$ and $\psi$, is it possible to convert $\phi$ to $\psi$ by LOCC? This task can be generalized naturally to a set of outcomes $\left\{\left(p_{i}, \psi_{i}\right)\right\}$, where each outcome $\psi_{i}$ is obtained with probability $p_{i}$.

Let us first see how this can be solved without SSR 13]. Therefore, let $\boldsymbol{\lambda}=\left(\lambda_{k}\right)$ and $\boldsymbol{\mu}^{i}=\left(\mu_{k}^{i}\right)$ be the Schmidt coefficients of $\phi$ and $\psi_{i}$, respectively, which completely characterize the states up to local unitaries. Without loss of generality, the Schmidt vectors may be taken decreasing $\left(\lambda_{k} \geq \lambda_{k+1}\right)$ and of equal dimension (by appending zeros). Following [13], an LOCC strategy for the conversion

$$
\phi \longrightarrow\left\{\left(p_{i}, \psi_{i}\right)\right\}
$$

exists if and only if (iff)

$$
\lambda \prec \sum_{i} p_{i} \boldsymbol{\mu}^{i} .
$$

Here, for two ordered vectors $\boldsymbol{\lambda}$ and $\boldsymbol{\mu}$, we say that $\boldsymbol{\lambda}$ is majorized by $\boldsymbol{\mu}, \boldsymbol{\lambda} \prec \boldsymbol{\mu}$, if $\sum_{k=1}^{d} \lambda_{k} \leq \sum_{k=1}^{d} \mu_{k}$ for all $1 \leq d<\operatorname{dim} \boldsymbol{\lambda}$, where equality holds for $d=\operatorname{dim} \boldsymbol{\lambda}$.

As an example, consider the states

$$
\begin{aligned}
& |\phi\rangle=\sqrt{\frac{1}{2}}|0\rangle_{A}|1\rangle_{B}+\sqrt{\frac{1}{2}}|1\rangle_{A}|0\rangle_{B} \text { and } \\
& |\psi\rangle=\sqrt{\frac{1}{3}}|0\rangle_{A}|1\rangle_{B}+\sqrt{\frac{2}{3}}|1\rangle_{A}|0\rangle_{B},
\end{aligned}
$$

which have the ordered Schmidt vectors $\boldsymbol{\lambda}=(1 / 2,1 / 2)$ and $\boldsymbol{\mu}=(2 / 3,1 / 3)$, respectively. Since $\boldsymbol{\lambda} \prec \boldsymbol{\mu}$, it is possible to convert $\phi \rightarrow \psi$; for instance, Alice might start with the POVM measurement given by $M_{1}=\sqrt{1 / 3}|0\rangle\langle 0|+$ $\sqrt{2 / 3}|1\rangle\langle 1|$ and $M_{2}=\sqrt{1 / 3}|0\rangle\langle 0|+\sqrt{2 / 3}| 1\rangle\langle 1|$ which yields the two states

$$
\begin{aligned}
& \left|\psi_{1}\right\rangle=\sqrt{\frac{1}{3}}|0\rangle_{A}|1\rangle_{B}+\sqrt{\frac{2}{3}}|1\rangle_{A}|0\rangle_{B} \text { and } \\
& \left|\psi_{2}\right\rangle=\sqrt{\frac{2}{3}}|0\rangle_{A}|1\rangle_{B}+\sqrt{\frac{1}{3}}|1\rangle_{A}|0\rangle_{B} .
\end{aligned}
$$

with equal probabilities: $\psi_{1}$ is already equal to $\psi$, and $\psi_{2}$ can be converted to $\psi$ by a bilateral NOT operation.

Let us now see what is different when SSR apply: while the POVM measurement $\left\{M_{1}, M_{2}\right\}$ is compatible with the superselection rule, the local application of NOT operations is not; indeed, it is not possible at all to carry out $\phi \rightarrow \psi$ deterministically in the presence of SSR. In order to see this, define block-diagonal POVM operators $M_{i}=\bigoplus_{n} M_{n}^{i}$ on one local system. Then, the completeness relation $\sum M_{i}^{\dagger} M_{i}=\mathbb{1}$ yields $\sum_{i} M_{n}^{i \dagger} M_{n}^{i}=\mathbb{1}$ for all $n$. Therefore, any POVM operator is simply a direct sum of POVM operators acting within the subspaces with constant local particle number, i.e., the usual conditions for convertibility have to hold for each subspace separately. Particularly, this implies that for pure states the average weight of each subspace with constant local particle number cannot be changed by local operations.

The impossibility to change the average weight of a subspace with fixed local particle number can even be proven at a much more fundamental level. Take multiple copies of some state $|\phi\rangle$ with nonconstant local particle number, and assume there is a way for Alice to change her local particle number distribution on average. As the total particle number is constant, this implies that the average particle number distribution of Bob's system is changed the other way round. Therewith, Alice can change Bob's density matrix remotely which would allow for supraluminal communication and therefore has to be ruled out. Classical communication between Alice and Bob, on the other hand, will increase Bob's knowledge of the actual particle number distribution, but it cannot influence the average distribution obtained.

In order to formulate this result precisely, note that any bipartite state $\phi \in \mathcal{H}_{N}$ subject to SSR can be written as $\phi=\phi^{0} \oplus \cdots \oplus \phi^{N}$ with $\phi^{n} \in \mathcal{H}_{n}^{A} \otimes \mathcal{H}_{N-n}^{B}$, i.e., as a direct sum of unnormalized pure states with constant local particle number. Call the (ordered) unnormalized Schmidt coefficients of $\phi^{n} \boldsymbol{\lambda}^{n}$. Then, $\phi$ is characterized up to local (SSR-compatible) unitaries by its $S S R$-ordered Schmidt vector $\boldsymbol{\lambda}=\left(\boldsymbol{\lambda}^{0}, \ldots, \boldsymbol{\lambda}^{N}\right)$.

Theorem 1 ([17]). Let $\phi, \psi_{i}$ be pure states and $\boldsymbol{\lambda}, \boldsymbol{\mu}_{i}$ their SSR-ordered Schmidt vectors. Then,

$$
\phi \stackrel{\mathrm{SSR}}{\longrightarrow}\left\{\left(p_{i}, \psi_{i}\right)\right\}
$$

(i.e., there exists a SSR-compatible conversion strategy) if and only if

$$
\lambda^{n} \prec \sum_{i} p_{i} \boldsymbol{\mu}_{i}^{n} \quad \forall n=0, \ldots, N .
$$

In order to see the connection to the conversions within the subspaces, let us re-express (5) by normalizing the SSR-ordered Schmidt vectors,

$$
\hat{\boldsymbol{\lambda}}^{n} \prec \sum_{i} \underbrace{p_{i} \frac{\left\|\boldsymbol{\mu}_{i}\right\|}{\|\boldsymbol{\lambda}\|}}_{=: p_{i}^{\prime}} \hat{\boldsymbol{\mu}}_{i}^{n} \quad \forall n=0, \ldots, N,
$$

where in the following a hat $\hat{\imath}$ denotes the normalized vector. According to the usual majorization result, this holds iff we can convert

$$
\hat{\phi}^{n} \longrightarrow\left\{p_{i}^{\prime}, \hat{\psi}_{i}^{n}\right\} \quad \forall n=0, \ldots, N .
$$


Here, $\phi=\phi^{0} \oplus \cdots \oplus \phi^{N}$ and $\psi_{i}=\psi_{i}^{0} \oplus \cdots \oplus \psi_{i}^{N}$.

Proof. Exactly as without SSR, the most general strategy consists of Alice performing a generalized measurement and communicating the result to Bob, who then applies a unitary operation depending on the measurement outcome; the proof [18] can be directly transferred.

We show (4) $\Leftrightarrow$ (6). The proof can be restricted to the case where each conversion $\phi \rightarrow(p, \psi)$ in (4) resp. (6) can be accomplished by a single POVM operator $M$, i.e., $M \phi=\sqrt{p} \psi$ - otherwise, we can split $\phi \rightarrow(p, \psi)$ into $\phi \rightarrow$ $\left(p_{k}, \psi\right), \sum p_{k}=p$, where each conversion is the result of one of the POVM operators. This can be done as well for the system of conversions (6), where we have to split all subspaces simultaneously (this can be always done by additionally splitting single POVM operators into copies of itself).

First, assume that (4) holds. Then there exist POVM operators $M_{i}=\bigoplus_{n} M_{i}^{n}$ on Alices side for which $M_{i} \phi \cong_{B}$ $\sqrt{p_{i}} \psi$ (i.e., up to a unitary on Bob's side). Decomposing this into the subspaces in the direct sum, one obtains $M_{i}^{n} \phi^{n} \cong_{B} \sqrt{p_{i}} \psi_{i}^{n}$ and thus

$$
M_{i}^{n} \hat{\phi}^{n} \cong \underbrace{\sqrt{p_{i} \frac{\left\langle\psi_{i} \mid \psi_{i}\right\rangle}{\langle\phi \mid \phi\rangle}}}_{\equiv \sqrt{p_{i}^{\prime}}} \hat{\psi}_{i}^{n}
$$

for all $N$, i.e., the $M_{i}^{n}$ accomplish the set of conversions given by Eq. (6). Especially, as

$$
\mathbb{1}=\sum_{i}\left(\bigoplus_{n} M_{i}^{n}\right)^{\dagger}\left(\bigoplus_{n} M_{i}^{n}\right)=\bigoplus_{n}\left(\sum_{i} M_{i}^{n \dagger} M_{i}^{n}\right),
$$

the $M_{i}^{n}$ obey the completeness relation for POVM operators. As all arguments hold in both directions, this completes the proof.

\section{B. Variance as a nonlocal monotone}

Let us now formulate an asymptotic version of the previous theorem. It is known that without SSR for a large number of copies the majorization criterion converges to the entropic criterion, i.e., the conservation of the total EoE [1]. With SSR, the probability distribution associated to the variation of the local particle number, $p_{n}=\sum_{i} p_{i}^{n}$, has to be conserved as well. Asymptotically, this distribution converges to a Gaussian which is completely characterized by its mean (which can be shifted using ancillas) and its variance. Therefore we define

Definition 1. For a bipartite pure state $\phi$ shared by $A$ and $B$, define the superselection induced variance (SiV)

$$
V(\phi):=4\left[\left\langle\phi\left|\hat{N}_{A}^{2}\right| \phi\right\rangle-\left\langle\phi\left|\hat{N}_{A}\right| \phi\right\rangle^{2}\right],
$$

where $N_{A}$ is the particle number operator for Alice. (One could equally well take $\hat{N}_{B}$, as $\hat{N}_{A}+\hat{N}_{B}=N=$ const.)
The factor 4 in the definition normalizes the $\mathrm{SiV}$ : $V(|\mathrm{~V}-\mathrm{EPR}\rangle)=1$.

Let us now show that $\mathrm{SiV}$ is really an entanglement monotone [19] when SSR are present, namely that it cannot be increased on average by SSR-LOCC and vanishes on separable states. (On the contrary, note that $V(\phi)=0$ does not imply that $\phi$ is separable - this is due to the fact that there exist two different nonlocal quantities when SSR are present.) Moreover, SiV is symmetric under interchange of $A$ and $B$ and additive: given two subsystems 1 and 2 shared by $A$ and $B, V\left(\phi_{1} \otimes \phi_{2}\right)=V\left(\phi_{1}\right)+V\left(\phi_{2}\right)$, as can be readily seen by applying Eq. (2) to the two subsystems 1 and 2, $\hat{N}_{A 1 A 2}=\hat{N}_{A 1} \otimes \mathbb{1}_{A 2}+\mathbb{1}_{A 1} \otimes \hat{N}_{A 2}$.

To show the monotonicity of SiV under SSR-LOCC, consider a POVM measurement $\left\{M_{i}^{A}\right\}$ on Alice's side. Then, the average $\mathrm{SiV}$ after the application of $\left\{M_{i}^{A}\right\}$ is given by

$\bar{V}_{M}(\phi)=\sum_{i}\left\langle\phi\left|M_{i}^{A \dagger} \hat{N}_{A}^{2} M_{i}^{A}\right| \phi\right\rangle-\sum_{i} \frac{\left\langle\phi\left|M_{i}^{A \dagger} \hat{N}_{A} M_{i}^{A}\right| \phi\right\rangle^{2}}{\left\langle\phi\left|M_{i}^{A \dagger} M_{i}^{A}\right| \phi\right\rangle}$.

The first part reduces to $\left\langle\phi\left|\hat{N}_{A}^{2}\right| \phi\right\rangle$ (using $\left[\hat{N}_{A}, M_{i}^{A}\right]=0$ and $\sum_{i} M_{i}^{A \dagger} M_{i}^{A}=\mathbb{1}$ ), while for the second part

$$
\begin{aligned}
\sum_{i} \frac{\left\langle\phi\left|M_{i}^{A \dagger} \hat{N}_{A} M_{i}^{A}\right| \phi\right\rangle^{2}}{\left\langle\phi\left|M_{i}^{A \dagger} M_{i}^{A}\right| \phi\right\rangle} & \stackrel{(*)}{\geq}\left(\sum_{i}\left\langle\phi\left|M_{i}^{A \dagger} \hat{N}_{A} M_{i}^{A}\right| \phi\right\rangle\right)^{2} \\
& =\left\langle\phi\left|\hat{N}_{A}\right| \phi\right\rangle^{2} .
\end{aligned}
$$

Here, $(*)$ has been derived using the Cauchy-Schwarz inequality

$$
\left(\sum_{i} y_{i}\right)^{2}=\left(\sum_{i} \sqrt{p_{i}} \frac{y_{i}}{\sqrt{p_{i}}}\right)^{2} \leq \sum_{i} \frac{y_{i}^{2}}{p_{i}} \sum_{i^{\prime}} p_{i^{\prime}} .
$$

Ancillas leave the result unaffected, as the extra contributions in $\bar{V}_{M}(\phi)$ originating from $[\hat{N}, \mathcal{O}]=\nu \mathcal{O}$ cancel out.

\section{Reversible conversion of multiple copies}

The introduction of $\mathrm{SiV}$ as a nonlocal monotone was motivated by the conversion of multiple copies, as it characterizes the joint particle number distribution. In the following, we will show that asymptotically SiV and EoE quantify the two complementary resources which completely characterize biparitite states up to SSR-LOCC.

Theorem 2. In the presence of SSR, there exists an asymtotically reversible conversion

$|\phi\rangle^{\otimes N} \otimes|\hat{0}\rangle^{\otimes E(\phi) N} \longleftrightarrow \sum_{n} c_{n}|n\rangle|N-n\rangle \otimes|\mathrm{E}-\mathrm{EPR}\rangle^{\otimes E(\phi) N}$,

where the coefficients $c_{n}$ are distributed Gaussian with

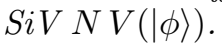


Note that on the left hand side we have added ancilla states in the unrestricted "hat"-basis (cf. Sec. III). The conversion transfers the entanglement contained in $|\phi\rangle^{\otimes N}$ to this second register as "accessible" entanglement in the form of $|\mathrm{E}-\mathrm{EPR}\rangle \mathrm{s}$, while the $\mathrm{SiV}$ stays in the first register.

Proof. First, we restrict ourself to the case of qubits, where $|\phi\rangle=\sqrt{p_{0}}|0\rangle|1\rangle+\sqrt{p_{1}}|1\rangle|0\rangle$. We will generalize the result in two steps: in a first step, we consider qu- $d$-its, where the local basis is $\{|0\rangle, \ldots,|d-1\rangle\}$, while in a second step we allow for arbitrary bipartite states, i.e., the local bases might contain several states with the same particle number.

For the beginning, let us only look at the first register. Taking $N$ copies of $|\phi\rangle$, we have

$$
|\phi\rangle^{\otimes N}=\sum_{\mathbf{x}} \sqrt{p_{0}^{n_{0}} p_{1}^{n_{1}}}|\mathbf{x}\rangle|\neg \mathbf{x}\rangle
$$

where the sum is taken over all possible $N$-bit strings $\mathbf{x}$. Here, $n_{0}$ and $n_{1}$ are the numbers of zeroes and ones in $\mathbf{x}$, respectively, and $\neg \mathbf{x}$ denotes the bitwise NOT of $\mathbf{x}$. This state can be grouped naturally as

$$
|\phi\rangle^{\otimes N}=\sum_{n_{0}} \sqrt{p_{0}^{n_{0}} p_{1}^{N-n_{0}}\left(\begin{array}{c}
N \\
n_{0}
\end{array}\right)}\left|\chi_{N-n_{0}, n_{0}}\right\rangle
$$

where the state $\left|\chi_{N-n_{0}, n_{0}}\right\rangle \in \mathcal{H}_{N-n_{0}}^{A} \otimes \mathcal{H}_{n_{0}}^{B}$ is a maximally entangled state with Schmidt number $\left(\begin{array}{c}N \\ n_{0}\end{array}\right)$.

In the following, we show how to transfer the entanglement of $|\phi\rangle^{\otimes N}$ to the second register. Therefore, we have to break the tensor product structure $|\phi\rangle^{\otimes N}$ of the first register and create a new tensor product structure by properly transferring the entanglement to the second register. To this end, let us introduce the concept of typical subspaces 20]. An $\epsilon$-typical subspace of our Hilbert space is defined as $\mathcal{H}_{\epsilon}=\bigoplus_{n_{0} \in \mathcal{S}_{\epsilon}} \mathcal{H}_{N-n_{0}}^{A} \otimes \mathcal{H}_{n_{0}}^{B}$, where the $\epsilon$-typical $n_{0}$ are those lying in $\mathcal{S}_{\epsilon}=\left\{n_{0}:\left|n_{0} / N-p_{0}\right|<\epsilon\right\}$. It can be shown [20, 21] that projecting $|\phi\rangle^{\otimes N}$ onto $\mathcal{H}_{\epsilon}$ gives an error which vanishes for $N \rightarrow \infty$ such that we can restrict the sum in (8) to $n_{0} \in \mathcal{S}_{\epsilon}$. Then,

$$
\left(\begin{array}{c}
N \\
n_{0}
\end{array}\right) \geq \frac{1}{(N+1)^{2}} 2^{N H\left(\frac{n_{0}}{N}\right)} \geq 2^{N\left[H\left(p_{0}\right)-K \epsilon\right]}
$$

with some $K>0$ holds for all $n_{0} \in \mathcal{S}_{\epsilon}(\epsilon \ll 1$ and $N \gg$ 1) 20]; here $H(p)=H(p, 1-p)$ is the Shannon entropy of the probability distribution $(p, 1-p)$. According to Theorem 1] we can transform

$\left|\chi_{N-n_{0}, n_{0}}\right\rangle \rightarrow \frac{1}{\sqrt{E}} \sum_{i=1}^{E}\left|i_{N-n_{0}}\right\rangle_{A}\left|i_{n_{0}}^{\prime}\right\rangle_{B} ; E=H\left(p_{0}\right)-K \epsilon$

coherently in all subspaces in the restricted sum, where $\left|i_{n}\right\rangle$ are orthogonal states with $n$ particles. Then by local maps $\left|i_{n}\right\rangle|\hat{0}\rangle \mapsto|n\rangle|\hat{i}\rangle$, where $|\hat{i}\rangle$ are orthogonal and $|n\rangle=|1 \cdots 10 \cdots 0\rangle$, the entanglement $H\left(p_{0}\right)-K \epsilon$ can be transferred to the second register which gives

$$
\sum_{n_{0} \in \mathcal{S}_{\epsilon}} c_{n_{0}}\left|N-n_{0}\right\rangle\left|n_{0}\right\rangle \otimes[|01\rangle|10\rangle+|10\rangle|01\rangle]^{\otimes N\left[H\left(p_{0}\right)-K \epsilon\right]}
$$

where $c_{n_{0}}=\sqrt{p_{0}^{n_{0}} p_{1}^{N-n_{0}}\left(\begin{array}{c}N \\ n_{0}\end{array}\right)}$. The sum can be extended to all $n_{0}$ with high fidelity, and the $\left|c_{n_{0}}\right|^{2}$ approach a Gaussian distribution with variance $N p_{0}\left(1-p_{0}\right)=$ $V(\phi) / 4$. This is the only parameter characterizing the state (10), since the mean can be shifted by locally adding ancillas. As $H\left(p_{0}\right)$ is just $E(\phi)$, this completes the distillation direction of the proof.

The dilution direction can be proven using the converse of (9),

$$
\left(\begin{array}{c}
N \\
n_{0}
\end{array}\right) \leq 2^{N H\left(\frac{n_{0}}{N}\right)} \leq 2^{N\left[H\left(p_{0}\right)+K \epsilon\right]}
$$

in an $\epsilon$-typical subspace. Starting from

$$
\sum_{n_{0} \in \mathcal{S}_{\epsilon}} c_{n_{0}}\left|N-n_{0}\right\rangle\left|n_{0}\right\rangle \otimes[|01\rangle|10\rangle+|10\rangle|01\rangle]^{\otimes N\left[H\left(p_{0}\right)+K \epsilon\right]},
$$

we can transfer the entanglement to the first register and then (again by Theorem 11) reduce the Schmidt number of each subspace to $\left(\begin{array}{c}N \\ n_{0}\end{array}\right)$, obtaining the projection of $|\phi\rangle^{\otimes N}$ onto the $\epsilon$-typical subspace, so that the dilution works as well. This completes the proof for qubits.

In a first step, we generalize the proof from qubits to $(I+1)$-level systems,

$$
|\phi\rangle=\sum_{i=0}^{I} \sqrt{p_{i}}|i\rangle|I-i\rangle
$$

(Note that the coefficients can be made positive by local operations.) Again, for $N$ copies of $|\phi\rangle$, an $\epsilon$-typical subspace can be defined by restricting the number $n_{i}$ of occurences of the state $|i\rangle|I-i\rangle$ in the product by $\left|n_{i} / N-p_{i}\right|<\epsilon$ for all $i$. Projecting the state onto an $\epsilon$-typical subspace again only yields a vanishingly small error, and the Schmidt number of the states with fixed numbers $\left(n_{0}, \ldots, n_{I}\right)$ is given by the multinomial coefficient $\left(\begin{array}{ccc} & N & \\ n_{0} & \cdots & n_{I}\end{array}\right)$ and obeys the bounds [20]

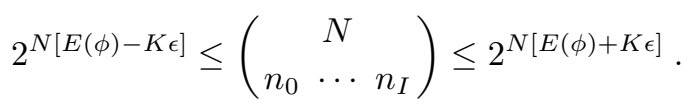

Thus, it is possible to extract the entanglement $E(\phi)$ reversibly. Yet, there are several possible configurations $\left(n_{0}, \ldots, n_{I}\right)$ which yield the same local particle number $n=\sum_{i} i n_{i}$ such that there is still some entanglement left in each subspace. But as for $N$ copies of $|\phi\rangle$ the number of these configurations is bounded by $N^{I}$, this entanglement is logarithmic in $N$ and can be removed reversibly. 


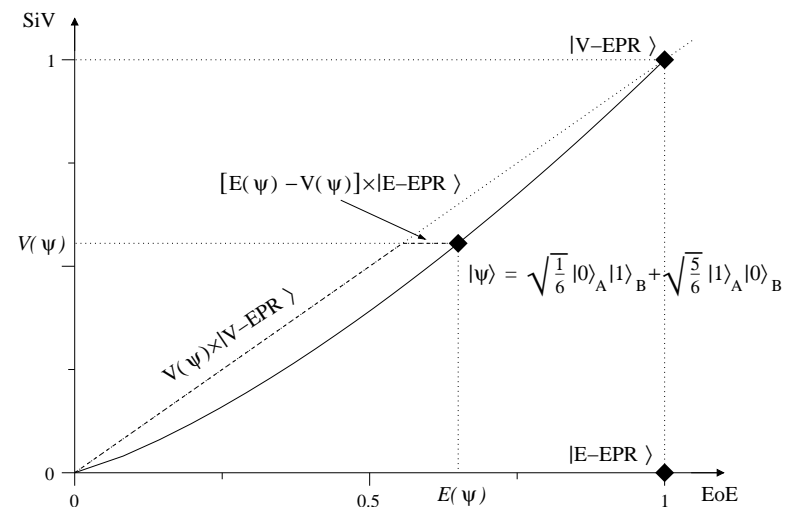

FIG. 1: Characterization of pure qubit states in an $E$ - $V$ diagram. All the states reside on the solid curve; asymptotically, any state can be converted into $V(\psi)$ copies of a $\mid \mathrm{V}$-EPR $\rangle$ and $E(\psi)-V(\psi)$ of a $|\mathrm{E}-\mathrm{EPR}\rangle$.

Therefore, we can reversibly transform $|\phi\rangle^{\otimes N} \otimes|\hat{0}\rangle^{\otimes N E(\phi)}$ into

$$
\sum c_{n}|n\rangle|I N-n\rangle \otimes[|01\rangle|10\rangle+|10\rangle|01\rangle]^{\otimes N E(\phi)},
$$

where the $c_{n}$ are given by the sum over all coefficients for which the particle number on Alice's side is $n$,

$$
c_{n}=\sqrt{\sum_{\substack{\sum_{i} i n_{i}=n \\
\sum_{i} n_{i}=N}} p_{0}^{n_{0}} \cdots p_{I}^{n_{I}}\left(\begin{array}{c}
N \\
n_{0} \cdots
\end{array}\right)} .
$$

It remains to be shown that the $\left|c_{n}\right|^{2}$ approach a Gaussian distribution. As long as all $p_{i} \neq 0$, this can be shown by expanding each $n_{i}$ within the typical subspace as $n_{i}=N\left(p_{i}+\delta_{i}\right)$ with $\delta_{i}<\epsilon$. This will work fine whenever $N p_{i} \gg 1$ and $\epsilon \ll p_{i}$ for all $i$. Yet, this condition cannot be satisfied if $p_{i}=0$ for some $i$. This might (but need not!) lead to a periodic gap in the distribution of the $\left|c_{n}\right|^{2}$, e.g., for $I=2, p_{0}=p_{2}=1 / 2$. In that case, $\left|c_{n}\right|^{2}=0$ for all odd $n$.

In principle, such a gap has to be considered as a third nonlocal characteristic of a bipartite state. Still, it can be removed easily. In the example given above the gap is readily removed by adding one $|\mathrm{V}-\mathrm{EPR}\rangle$, such that the fraction of $\mid \mathrm{V}$-EPR $\rangle$ per copies of $|\phi\rangle$ vanishes. By further adding an $|\mathrm{E}-\mathrm{EPR}\rangle$ (those are obtained anyway in the distillation) the $|\mathrm{V}-\mathrm{EPR}\rangle$ can be re-obtained - it therefore merely acts as a catalyst, "freeing" the subspaces with odd particle number.

The generalization to an arbitrary state is straightforward. Take

$$
|\phi\rangle=\sum_{i=0}^{I} \sqrt{p_{i}}\left|\psi_{i, I-i}\right\rangle,
$$

where $\left|\psi_{i, I-i}\right\rangle \in \mathcal{H}_{i}^{A} \otimes \mathcal{H}_{I-i}^{B}$ might themselves be entangled states. Applying the concept of typical subspaces to Eq. (13), we find that the number of occurences of each

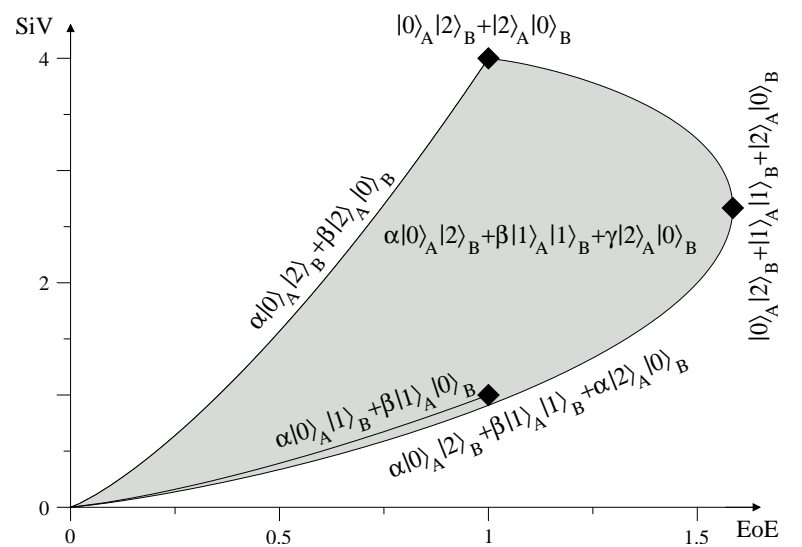

FIG. 2: $\quad E-V$ diagram for qutrits, where the boundary states and the extremal states are given. The possible states reside in the gray area, the solid line within this area is the subset realizable by qubits.

$\left|\psi_{i, I-i}\right\rangle$ in the typical subspace is bounded by $\left(p_{i} \pm \epsilon\right) N$, and thus the entanglement $E\left(\psi_{i, I-i}\right)$ contained in these states - which is already "accessible entanglement" - can be extracted reversibly. The remaining state is of the type of Eq. (11) (with the same coefficients $p_{i}$ ) and thus can be transformed reversibly into a Gaussian distributed state with width $N V(\phi)$ and $N H\left(p_{0}, \ldots, p_{I}\right)$ ebits of entanglement. It can be checked easily that the total number of Bell pairs is $N E(\phi)$.

For qubits, Theorem 2 can be re-expressed.

Corollary 1. For bipartite qubit states $|\phi\rangle$,

$$
|\phi\rangle \longleftrightarrow|\mathrm{E}-\mathrm{EPR}\rangle^{\otimes[E(\phi)-V(\phi)]}|\mathrm{V}-\mathrm{EPR}\rangle^{\otimes V(\phi)}
$$

in the asymptotic limit.

This can be shown by applying Theorem 2 twice, together with $E(\phi) \geq V(\phi)$ (which only holds for qubits).

Fig. 1 illustrates this characterization of states in the $E-V$ diagram. Fig. 2 shows the $E-V$ diagram for qutrits, which is considerable more complex. The bounds are given by the states with highest variance $\alpha|0\rangle_{A}|2\rangle_{B}+\beta|2\rangle_{A}|0\rangle_{B}$ and the states with highest entanglement $\alpha|0\rangle_{A}|2\rangle_{B}+\beta|1\rangle_{A}|1\rangle_{A}+\alpha|0\rangle_{A}|2\rangle_{B}$. A decomposition as in the Corollary is still possible if one replaces the $\mid \mathrm{V}$-EPR $\rangle$ by $|0\rangle_{A}|2\rangle_{B}+|2\rangle_{A}|0\rangle_{B}$ which has maximal variance.

\section{MIXED STATES IN THE PRESENCE OF SUPERSELECTION RULES}

\section{A. Introduction}

In the following section, we consider mixed states. We will show how the concepts of EoE and SiV as two complementary resources can be extended to mixed states, 
and discuss the connection with normal (SSR-free) entanglement measures.

Let us start by introducing a particularly interesting mixed state,

$$
\rho_{\text {sep }}=\frac{1}{4}\left(\begin{array}{cccc}
1 & & & \\
& 1 & 1 & \\
& 1 & 1 & \\
& & & 1
\end{array}\right)
$$

in the basis $\left\{|0\rangle_{A}|0\rangle_{B},|0\rangle_{A}|1\rangle_{B},|1\rangle_{A}|0\rangle_{B},|1\rangle_{A}|1\rangle_{B}\right\}$. This state has first been considered in [], where it was shown that it is separable but nonlocal. Namely, it can be obtained by mixing $\left(|0\rangle_{A}+\omega|1\rangle_{A}\right)\left(|0\rangle_{B}+\omega|1\rangle_{B}\right)$ for $\omega \in\{1, i,-1,-i\}$ with equal probabilities and therefore does not contain EoE. On the other hand, it is easy to see that there is no decomposition of $\rho_{\text {sep }}$ which is separable and compatible with the superselection rule, i.e., it cannot be created locally. Clearly, this can not happen with pure states.

Considering the results of the preceding section, it is natural to assume that $\rho_{\text {sep }}$ contains $\mathrm{SiV}$ but no EoE. In order to give quantitative meaning to such statements, we discuss two genuine extensions of nonlocal quantities to mixed states, defined by the asymptotic amount of pure state resources which are needed to create them and which can be extracted again.

\section{B. Formation of mixed states}

Let us start with the creation of mixed state in the presence of SSR. Similar to the normal case [14], we define:

Definition 2. The entanglement of formation and the variance of formation in the presence of superselection rules are defined as

$$
E_{F}^{\mathrm{SSR}}(\rho)=\min _{\left\{p_{i}, \psi_{i}\right\}} \sum_{i} p_{i} E\left(\psi_{i}\right)
$$

and

$$
V_{F}^{\mathrm{SSR}}(\rho)=\min _{\left\{p_{i}, \psi_{i}\right\}} \sum_{i} p_{i} V\left(\psi_{i}\right),
$$

respectively. The minimum is taken over all possible decompositions of $\rho$, where the $\psi_{i}$ have to obey the SSR (i.e., they all have constant particle number).

The entanglement cost [2\%] and the variance cost in the presence of superselection rules are accordingly defined as the regularized versions of $E_{F}^{\mathrm{SSR}}$ and $V_{F}^{\mathrm{SSR}}$,

$$
E_{c}^{\mathrm{SSR}}(\rho)=\lim _{N \rightarrow \infty} \frac{E_{F}^{\mathrm{SSR}}\left(\rho^{\otimes N}\right)}{N}
$$

and

$$
V_{c}^{\mathrm{SSR}}(\rho)=\lim _{N \rightarrow \infty} \frac{E_{F}^{\mathrm{SSR}}\left(\rho^{\otimes N}\right)}{N} .
$$

These definitions make sense, as they quantify the nonlocal resources we need at least to prepare the state $\rho$ with SSR 22.

As shown at the beginning of the section there exist states which do not contain any entanglement yet are nonlocal, as $\rho_{\text {sep }}$ [Eq. (14)]. One easily finds that $E_{F}^{\mathrm{SSR}}\left(\rho_{\text {sep }}\right)=1 / 2, V_{F}^{\mathrm{SSR}}\left(\rho_{\text {sep }}\right)=1 / 2$, as each of the subblocks in $\rho_{\text {sep }}$ has to be created separately. On the other hand, it seems reasonable to assume that $\rho_{\text {sep }}$ can be prepared asymptotically without using entanglement. In the following, we prove an even stronger result: asymptotically, the entanglement needed to create any state $\rho$ is just the entanglement needed without SSR.

Theorem 3. For any $\rho$ with bounded maximal particle number,

$$
E_{c}^{\mathrm{SSR}}(\rho)=E_{c}(\rho),
$$

i.e., the entanglement cost with SSR is the entanglement cost without SSR.

Proof. Consider a mixed state $\sigma$ compatible with the SSR and let $\sum_{i} p_{i}\left|\psi_{i}\right\rangle\left\langle\psi_{i}\right|=\sigma$ be the optimal decomposition without SSR, i.e., $E_{F}(\rho)=\sum_{i} p_{i} E\left(\psi_{i}\right)$. Clearly, this decomposition need not obey the SSR, but we can use it to constuct a compatible decomposition with vanishing overhead. From (11), $\sigma=\sum_{n=0}^{N} P_{n} \sigma P_{n}$, where $P_{n}$ is the projector onto the subspace with totally $n$ particles and $N$ the maximum total particle number in $\sigma$; therefore,

$$
\sigma=\sum_{n, i} p_{i} p_{i, n} \frac{P_{n}\left|\psi_{i}\right\rangle\left\langle\psi_{i}\right| P_{n}}{p_{i, n}}
$$

with $p_{i, n}=\left\langle\psi_{i}\left|P_{n}\right| \psi_{i}\right\rangle$ is a decomposition of $\sigma$ which is compatible with the SSR. For any $|\psi\rangle$ with at most $N$ particles, it holds that the measurement of the total particle number creates at most $\log (N+1)$ entanglement on average,

$$
\sum_{n}\left\langle\psi\left|P_{n}\right| \psi\right\rangle E\left(\frac{P_{n}|\psi\rangle}{\sqrt{\left\langle\psi\left|P_{n}\right| \psi\right\rangle}}\right) \leq E(|\psi\rangle)+\log (N+1)
$$

(the proof is given in the appendix), and with $\sigma=\rho^{\otimes M}$, the claim follows.

Note that this also implies that $E_{F}^{\mathrm{SSR}}$ is not additive [8]; $E_{F}^{\mathrm{SSR}}\left(\rho_{\mathrm{sep}}^{\otimes N}\right)$, e.g., grows at most logarithmically.

Let us now consider $V_{F}^{\mathrm{SSR}}$ and $V_{c}^{\mathrm{SSR}}$. As expected, the entanglement cost of $\rho_{\text {sep }}$ vanishes. But as $\rho_{\text {sep }}$ still contains some kind of nonlocality, it is natural to assume that its variance cost is strictly nonzero. In the following, we prove a more general result, namely that $V_{F}^{\mathrm{SSR}}$ is additive on all states which are a direct sum of pure states (i.e., $\rho$ is block-diagonal and each block is a pure state); this holds, e.g., for $\rho_{\text {sep }}$.

Theorem 4. Let $\rho=\bigoplus_{i} p_{i}\left|\phi_{i}\right\rangle\left\langle\phi_{i}\left|, \sigma=\bigoplus_{j} q_{j}\right| \psi_{j}\right\rangle\left\langle\psi_{j}\right|$, where $\sum_{i} p_{i}=\sum_{j} q_{j}=1$. Then

$$
V_{F}^{\mathrm{SSR}}(\rho \otimes \sigma)=V_{F}^{\mathrm{SSR}}(\rho)+V_{F}^{\mathrm{SSR}}(\sigma) .
$$


Proof.

$$
\begin{aligned}
V_{F}^{\mathrm{SSR}}(\rho \otimes \sigma) & =V_{F}^{\mathrm{SSR}}\left(\bigoplus_{i, j} p_{i} q_{j}\left|\phi_{i}\right\rangle\left\langle\phi_{i}|\otimes| \psi_{j}\right\rangle\left\langle\psi_{j}\right|\right) \\
& \stackrel{(*)}{=} \sum_{i, j} p_{i} q_{j} V\left(\phi_{i} \otimes \psi_{j}\right) \\
& =\sum_{i} p_{i} V\left(\phi_{i}\right)+\sum_{j} q_{j} V\left(\psi_{j}\right) \\
& \stackrel{(*)}{=} V_{F}^{\mathrm{SSR}}(\rho)+V_{F}^{\mathrm{SSR}}(\sigma),
\end{aligned}
$$

where in $(*)$ we used the equality $V_{F}^{\mathrm{SSR}}\left(\bigoplus_{i} r_{i}\left|\chi_{i}\right\rangle\left\langle\chi_{i}\right|\right)=$ $\sum_{i} r_{i} V\left(\chi_{i}\right)$ with $\sum_{i} r_{i}=1$. As subadditivity is clear from the convexity of $V_{F}^{\mathrm{SSR}}$, we only have to show superadditivity. For an arbitrary decomposition $\left|\zeta_{j}\right\rangle=$ $\sum_{i} u_{j i} \sqrt{r_{i}}\left|\chi_{i}\right\rangle$ of $\bigoplus_{i} r_{i}\left|\chi_{i}\right\rangle\left\langle\chi_{i}\left|=\sum_{j}\right| \zeta_{j}\right\rangle\left\langle\zeta_{j}\right|[$ with an isometry $\left.\left(u_{j i}\right)\right]$, this follows from

$$
\begin{aligned}
& \sum_{j} \frac{\left\langle\zeta_{j}\left|\hat{N}_{A}\right| \zeta_{j}\right\rangle^{2}}{\left\langle\zeta_{j} \mid \zeta_{j}\right\rangle} \stackrel{(\mathrm{a})}{=} \sum_{j} \frac{\left(\sum_{i} u_{j i}^{*} u_{j i} p_{i}\left\langle\chi_{i}\left|\hat{N}_{A}\right| \chi_{i}\right\rangle\right)^{2}}{\sum_{i} u_{j i}^{*} u_{j i} p_{i}} \\
& \stackrel{\text { (b) }}{=} \sum_{i, j} \frac{\left(u_{j i}^{*} u_{j i} p_{i}\left\langle\chi_{i}\left|\hat{N}_{A}\right| \chi_{i}\right\rangle\right)^{2}}{u_{j i}^{*} u_{j i} p_{i}} \stackrel{(\mathrm{c})}{=} \sum_{i} p_{i}\left\langle\chi_{i}\left|\hat{N}_{A}\right| \chi_{i}\right\rangle^{2} .
\end{aligned}
$$

Here, we used (a) $\left\langle\chi_{i} \mid \chi_{i^{\prime}}\right\rangle=\delta_{i i^{\prime}},\left\langle\chi_{i}\left|\hat{N}_{A}\right| \chi_{i^{\prime}}\right\rangle \propto \delta_{i i^{\prime}}$; (b) Eq. (7); (c) $\sum_{j} u_{j i}^{*} u_{j i}=1$.

While it seems plausible that $V_{F}^{\mathrm{SSR}}$ is additive on all states and we did not find any counterexamples, this is apparently hard to prove. Let us note that unlike for $E_{F}$, the additivity of $V_{F}^{\mathrm{SSR}}$ is probably not related to its superadditivity. A counterexample for the superadditivity of $V_{F}^{\mathrm{SSR}}$ can easily be found 23], and the direct equivalence proof of Pomeransky 24] cannot be transferred to $\mathrm{SiV}$ due to the different structure of the nonlinearity.

\section{Formation of qubits}

In the following, we compute explicit formulas for $E_{F}^{\mathrm{SSR}}$ and $V_{F}^{\mathrm{SSR}}$ of qubits. A general bipartite two-qubit state subject to SSR is given by

$$
\rho=\left(\begin{array}{cccc}
w_{00} & & & \\
& w_{01} & \gamma & \\
& \gamma & w_{10} & \\
& & & w_{11}
\end{array}\right)
$$

where $\gamma \geq 0$ (this can be achieved by local unitaries). Using the results of Wootters [25], we find $E_{F}(\rho)=\mathcal{E}(C)$, where $\mathcal{E}(C)=H\left(1 / 2+\sqrt{1-C^{2}} / 2\right), H$ is the binary entropy, and the concurrence $C \equiv C(\rho)$ can be computed as

$$
C=\max \left(0,2 \gamma-2 \sqrt{w_{00} w_{11}}\right)
$$

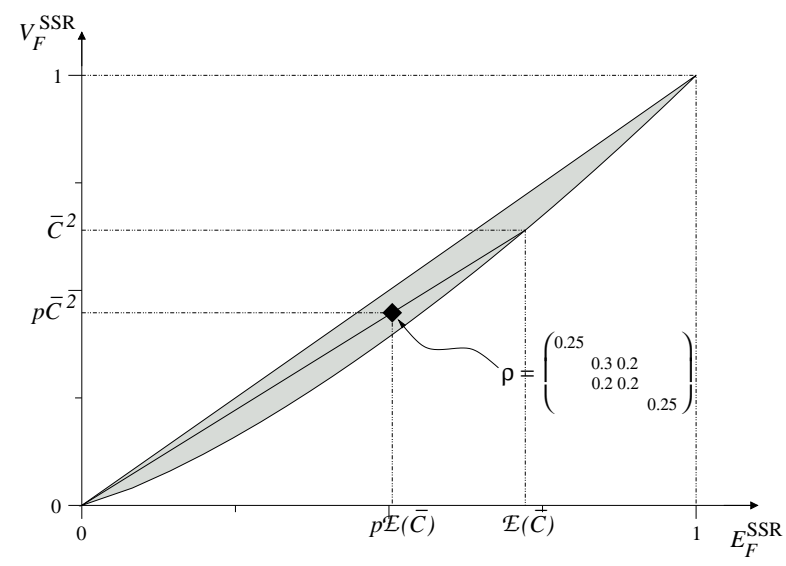

FIG. 3: Relation of $p, \bar{C}, E_{F}^{\mathrm{SSR}}$ and $V_{F}^{\mathrm{SSR}}$ (see Section IVC). The gray area gives the allowed range of $E_{F}^{\mathrm{SSR}}$ and $V_{F}^{\mathrm{SSR}}$ for qubits. The lower bound is obtained by plotting $\mathcal{E}(\bar{C})$ vs. $\bar{C}^{2}$. The point characterizing a mixed state $\rho$ can be found by dividing the line between the origin and the point $\left(\mathcal{E}(\bar{C}), \bar{C}^{2}\right)$ located on the boundary at the ratio of $p: 1-p$.

With SSR, $\rho$ has to be built subspace by subspace, where the one-particle subspace $\rho_{1}$ is the only one which might be entangled. The concurrence for $\rho_{1} / \operatorname{tr}\left[\rho_{1}\right]$ is

$$
\bar{C}=2 \gamma / p
$$

with $p=w_{01}+w_{10}=\operatorname{tr}\left[\rho_{1}\right]$, and thus

$$
E_{F}^{\mathrm{SSR}}(\rho)=p \mathcal{E}(\bar{C}) .
$$

The relation between the normal concurrence $C$ and the SSR-concurrence $\bar{C}$ is given by the bounds $p \bar{C}-(1-$ $p) \leq C \leq p \bar{C}$, i.e., $E_{F}$ and $E_{F}^{\mathrm{SSR}}$ are not completely independent. As $\mathcal{E}$ is concave, $E_{F} \leq E_{F}^{\mathrm{SSR}}$, as necessary.

An optimal decomposition of $\rho_{1}$ can be found as follows. Define $s$ as a root of $\bar{C} / 2=\sqrt{s(1-s)}$. Then, $\rho_{1}$ can be written as a mixture of $\sqrt{s}|01\rangle+\sqrt{1-s}|10\rangle$ and $\sqrt{1-s}|01\rangle+\sqrt{s}|10\rangle$, and both have the desired EoE.

The same decomposition gives the optimal variance as well (note that this only holds for qubits). Therefore, observe that both states have $\operatorname{SiV} 4 s(1-s)=\bar{C}^{2}$, i.e., $\bar{C}^{2}$ is an upper bound for $V_{F}^{\mathrm{SSR}}(\rho)$, and for pure states equality holds. On the other hand, $\bar{C}^{2}$ is convex: for any one-particle subblock $\rho_{1}=p \sigma+(1-p) \sigma^{\prime}$ with offdiagonal elements $v=p w+(1-p) w^{\prime}$ it holds that $v^{2} \leq$ $p w^{2}+(1-p) w^{\prime 2}$. Therefore equality holds, and

$$
V_{F}^{\mathrm{SSR}}(\rho)=p \bar{C}^{2} .
$$

Thus, with respect to formation $1 \times 1$ qubit states are characterized by two parameters: the weight of $\rho_{1}, p$, and the concurrence of $\rho_{1}, \bar{C}$. It can be checked easily that $0 \leq p, \bar{C} \leq 1$ in order for $\rho$ to be positive. A necessary condition for separable states is $\bar{C} \leq(1-p) / p$ (this is tight, but $p, \bar{C}$ do not tell everything about separability, cf. the inequality relating $C, \bar{C}$ given above).

Fig. [3] shows how for a particular state $p$ and $\bar{C}$ can be determined from the $E-V$ diagram, and Fig. 4 gives a "phase diagram" for mixed states. 


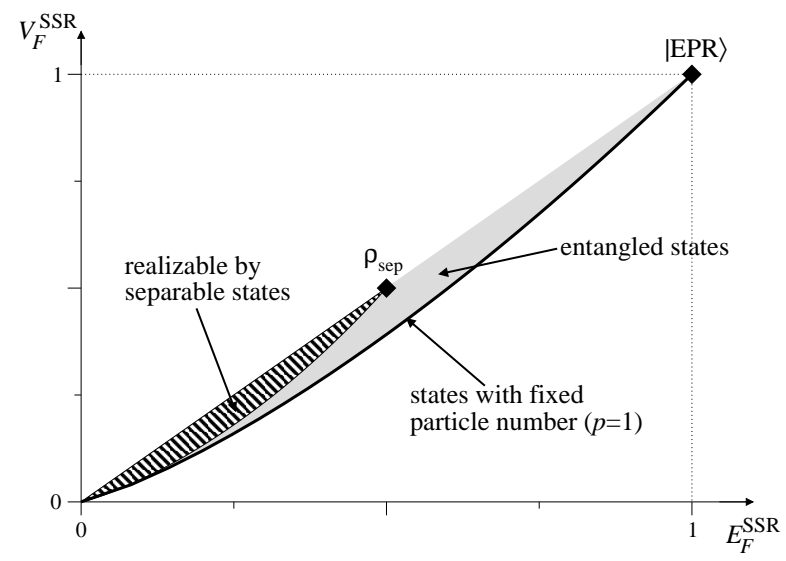

FIG. 4: Different regions of mixed states in the $E-V$ diagram. The solid line corresponds to the states with fixed total particle number, i.e., $p=1$. Separable states have to stay within the dashed area (although there exist entangled states as well). Note that $\rho_{\text {sep }}[\mathrm{Eq}$. 14] is the "extremal separable state".

\section{Distillation of nonlocal resources}

In the following, we consider the problem complementary to formation: given a mixed state, is it possible to distill the nonlocal resources contained in this state? This distilled state could then be used to perform some nonlocal task as teleportation with high fidelity. Naturally, there exist two types of distillation protocols: the first one aims to increase the fidelity of the states being distilled with the final state, while the second returns the target state itself with some finite yield in the asymptotic limit.

In the following, we will focus on qubits. Without SSR, it has been shown for both types of distillation how they can be implemented: in the so-called recurrence protocol [15], both parties apply an XOR operation (a bilateral XOR or BXOR) to two copies of the state and then measure one of them; thus, on average they increase their knwoledge about the second. Hashing protocols [14], on the contrary, are aimed to asymptotically return a finite yield of pure states: by subsequent application of BXOR operations partial information about the states can be collected in a subset which is then measured; by the law of large numbers, this partial information asymptotically fully determines the remaining states.

The presence of superselection rules inposes severe restrictions on distillation procedures. It has been shown, e.g., that the entanglement contained in one copy of a $\mid \mathrm{V}$-EPR $\rangle$ cannot be accessed, while for multiple copies of $\mid \mathrm{V}$-EPR $\rangle$, all entanglement up to a logarithmic amount can be used 8], as also follows from Thm. 2 The central problem in distilling states containing $\mathrm{SiV}$ is that the BXOR operation is ruled out by the SSR, and there is no adequate replacement. One way to overcome this problem is to use a third copy of the state as a (imperfect) shared reference frame and to construct a three-copy pro- tocol which probabilistically implements BxOR. Indeed, we will show that one needs three-copy protocols to distill both EoE and SiV. Unfortunately, this is of no use for the implementation of hashing protocols, as the errors of the BXOR-approximation accumulate, and each BXOR uses up the reference frame copy of the state whereas hashing would require $O\left(N^{2}\right)$ BXOR operations.

The existence of two distinct resources makes the field of distillation much more rich: there will occur trade-offs between the two resources in distillation, and one might even think of spending one resource to distill the other. For instance, we will show that it is possible to distill all separable but nonlocal states towards $\rho_{\text {sep }}$ [Eq. (14)], and in turn, if one adds some entanglement, all the $\mathrm{SiV}$ contained in $\rho_{\text {sep }}$ can be converted to a $\mid \mathrm{V}$-EPR $\rangle$.

\section{Reduction to standard states}

To simplify analysis, in 14 the distillation of qubits has been considered for a standard form, namely Belldiagonal states; any state can be made Bell-diagonal by LOCC. Yet, these operations are ruled out by the SSR, so that we have to introduce a different normal form. Therefore, consider a general bipartite qubit state with SSR

$$
\rho=\left(\begin{array}{cccc}
w_{00} & & & \\
& w_{01} & \gamma & \\
& \gamma^{*} & w_{10} & \\
& & & w_{11}
\end{array}\right),
$$

where $w_{i j} \geq 0$ and $\gamma \geq 0$ (the latter can be accomplished by local unitaries). By local filtering operations [26] $F_{A} \propto$ $\sqrt[4]{w_{10} w_{11}}|0\rangle\left\langle 0\left|+\sqrt[4]{w_{00} w_{01}}\right| 1\right\rangle\left\langle 1\left|, F_{B} \propto \sqrt[4]{w_{01} w_{11}}\right| 0\right\rangle\langle 0|+$ $\sqrt[4]{w_{00} w_{10}}|1\rangle\langle 1|$, this can be transformed probabilistically to

$$
\tilde{\rho}=\frac{1}{2(1+w)}\left(\begin{array}{cccc}
w & & & \\
& 1 & v & \\
& v & 1 & \\
& & & w
\end{array}\right) .
$$

Here, $w=\sqrt{\frac{w_{00} w_{11}}{w_{01} w_{10}}}$ and $v=\frac{|\gamma|}{\sqrt{w_{01} w_{10}}}$. In the following, we will call this the standard form $\tilde{\rho}$ of a two-qubit state $\rho$ and only consider states of this type. The standard form is Bell-diagonal and unique for each $\rho$, and by the reverse $\mathrm{POVM} F_{A}^{\prime} \propto F_{A}^{-1}, F_{B}^{\prime} \propto F_{B}^{-1}, \tilde{\rho}$ is converted back to $\rho$. Thus, any state can be transformed probabilistically to its standard form and back by LOCC, and therefore the standard form of states containing $\mathrm{EoE}$ and $\mathrm{SiV}$ still contains EoE and SiV [27].

Note that the two parameters $(w, v)$ describing the standard form $\tilde{\rho}$ are directly related to $(p, \bar{C})$ used to characterize $E_{F}^{\mathrm{SSR}}(\tilde{\rho})$ and $V_{F}^{\mathrm{SSR}}(\tilde{\rho})$ in Section IVC $v=$ $\bar{C}$ and $w=1 / p-1$. 


\section{Distilling entanglement}

Let us first demonstrate that it is possible to distill all entangled qubit states, as it is the case without SSR. Therefore, take two copies of an arbitrary state $\rho$ in its standard form $\tilde{\rho}$ [Eq. (18)] and project locally onto the one-particle subspaces. The resulting state in the $\{|\hat{0}\rangle,|\hat{1}\rangle\}$-basis is

$$
\hat{\rho}=\left(\begin{array}{cccc}
w^{2} & & & \\
& 1 & v^{2} & \\
& v^{2} & 1 & \\
& & & w^{2}
\end{array}\right)
$$

Obviously, $\hat{\rho}$ is entangled iff $\tilde{\rho}$ is entangled iff $\rho$ is entangled, and as $\hat{\rho}$ has constant local particle number, it can be distilled as usual [14, 15]. Therefore, it is possible to distill any entangled two-qubit state if we do not care about its SiV. Even more, if we have an infinite amount of $\mathrm{SiV}$ available, we can distill with the same rate as without SSR by using the SiV as a perfect reference frame.

\section{Distilling separable states}

Clearly, the SiV contained in separable but nonlocal states cannot be distilled, as pure states with SiV always contain entanglement. One solution to this problem is to distill towards $\rho_{\text {sep }}[\mathrm{Eq}$. (14)]; we will show how this can be done (and why $\rho_{\text {sep }}$ is a good choice) in the next subsection. Alternatively, one might try to add entanglement (e.g., $\mid$ E-EPR $\rangle$ s) and then distill the SiV of separable states to $|\mathrm{V}-\mathrm{EPR}\rangle \mathrm{s}$.

In the following, we show how $\rho_{\text {sep }} \otimes|\mathrm{E}-\mathrm{EPR}\rangle\langle\mathrm{E}-\mathrm{EPR}|$ can be transformed to a $\mid \mathrm{V}$-EPR $\rangle$ with probability $1 / 2$, thereby distilling all the $\mathrm{SiV}$ contained in $\rho_{\text {sep }}$ to a pure state. Clearly, $|\mathrm{V}-\mathrm{EPR}\rangle$ can be obtained from an $|\mathrm{E}-\mathrm{EPR}\rangle=|01\rangle|10\rangle+|10\rangle|01\rangle$ by applying a BXOR operation, but this is ruled out by the SSR. The idea in the following is to use the $\mathrm{SiV}$ contained in $\rho_{\text {sep }}$ as a shared reference frame in order to carry out the BXOR operation probabilistically. In order to see how this works, write $\rho_{\text {sep }}$ as a mixture of $(|0\rangle+\omega|1\rangle)_{A}(|0\rangle+\omega|1\rangle)_{B}$ over all $\omega=e^{i \phi}$. If we manage to project the total state onto subspaces where $\omega$ simply gives a global phase, we can make use of the $\mathrm{SiV}$ of $\rho_{\text {sep. }}$. Therefore, start with the state $|\mathrm{E}-\mathrm{EPR}\rangle\langle\mathrm{E}-\mathrm{EPR}| \otimes \rho_{\text {sep }}$ which can be written as a mixture of the states

$\left|\psi_{0}\right\rangle \propto|010\rangle|100\rangle+|100\rangle|010\rangle$,

$\left|\psi_{1}\right\rangle \propto|010\rangle|101\rangle+|100\rangle|011\rangle+|011\rangle|100\rangle+|101\rangle|010\rangle$,

$\left|\psi_{2}\right\rangle \propto|011\rangle|101\rangle+|101\rangle|011\rangle$

with probabilities $1 / 4,1 / 2$, and $1 / 4$. Clearly, there is no measurement which tells us which $\left|\psi_{i}\right\rangle$ we actually have without either destroying the entanglement contained in $\left|\psi_{0}\right\rangle$ and/or $\left|\psi_{2}\right\rangle$ or the variance contained in $\left|\psi_{1}\right\rangle$. As we want to extract the variance, we have to sacrifice the EoE of $\left|\psi_{0,2}\right\rangle$ : both parties do a projective measurement onto the subspaces spanned by $\{|010\rangle,|101\rangle\}$ and $\{|100\rangle,|011\rangle\}$. If the measurement outcomes match, Alice and Bob share a known state with EoE and SiV 1 which can be converted to a $\mid \mathrm{V}$-EPR $\rangle$; otherwise, the entanglement is lost. Both cases are equally likely, and thus the average yield of $\mathrm{SiV}$ is $1 / 2=V_{F}^{\mathrm{SSR}}\left(\rho_{\text {sep }}\right)$ which is optimal. On the other hand, we had to sacrifice half of the entanglement - there is a trade-off between the two resources.

The procedure described above can be generalized to arbitrary states, where it allows to distill the one-particle subblock. Note that if $\rho$ is entangled, the required $|\mathrm{E}-\mathrm{EPR}\rangle \mathrm{s}$ can be distilled from $\rho$ itself.

\section{Recurrence protocols}

In the following, we will look for protocols which allow to distill EoE and SiV. Particularly, we would like to have a protocol which allows to concentrate the SiV contained in separable states. As already mentioned at the beginning of the section, the usual recurrence protocols cannot be applied as BXOR cannot be implemented. (In fact, it is not even possible to find an operation doing a comparable job, i.e., computing the parity, only for $|0\rangle|1\rangle \pm|1\rangle|0\rangle$.) Yet, similar to the preceding subsection we can use a third copy as a shared reference frame which allows to implement the desired recurrence operation in a probabilistic way. Indeed, we will see that three-copy protocols suffice for all distillation tasks.

General $N$-copy recurrence protocols can be represented by local POVM operators which act on $N$ qubits and leave one qubit (i.e., $2 \times 2^{N}$ matrices). These operators must be realizable by SSR-compatible operations, i.e., by an $N$-qubit POVM, followed by a measurement of all but one qubit (omitting the measurement decreases our information about the state and thus does not help). Therefore, the POVM operators must have the shape of two adjacent rows of SSR-compatible $N$-qubit operations [Eq. (1)]; except normalization, this is the only condition.

Possible protocols are illustrated in Fig. [5] Any state can be brought to standard form Eq. (18) by local filtering operations and can be parametrized by a tuple $(v, w)$, $0 \leq v \leq 1,0 \leq w$; the states with $v>w$ are entangled (Fig. 5 a).

Given a single copy of $\tilde{\rho}(v, w)$, Alice and Bob can either increase $w$ (by adding $|00\rangle\langle 00|+| 11\rangle\langle 11|$ ) or decrease $v$ and $w$ by the same fraction (by adding $|01\rangle\langle 01|+$ $|10\rangle\langle 10|)$, and anything inbetween, as is illustrated in Fig. 5 b. Obviously, $\rho_{\text {sep }}$ can be transformed to any other separable state deterministically - therefore, it is indeed the standard separable but nonlocal state, as an EPR is for entanglement.

Let us turn our attention to two-copy protocols. As the output state will not necessarily have standard form, we have to include filtering in the local POVM operators 

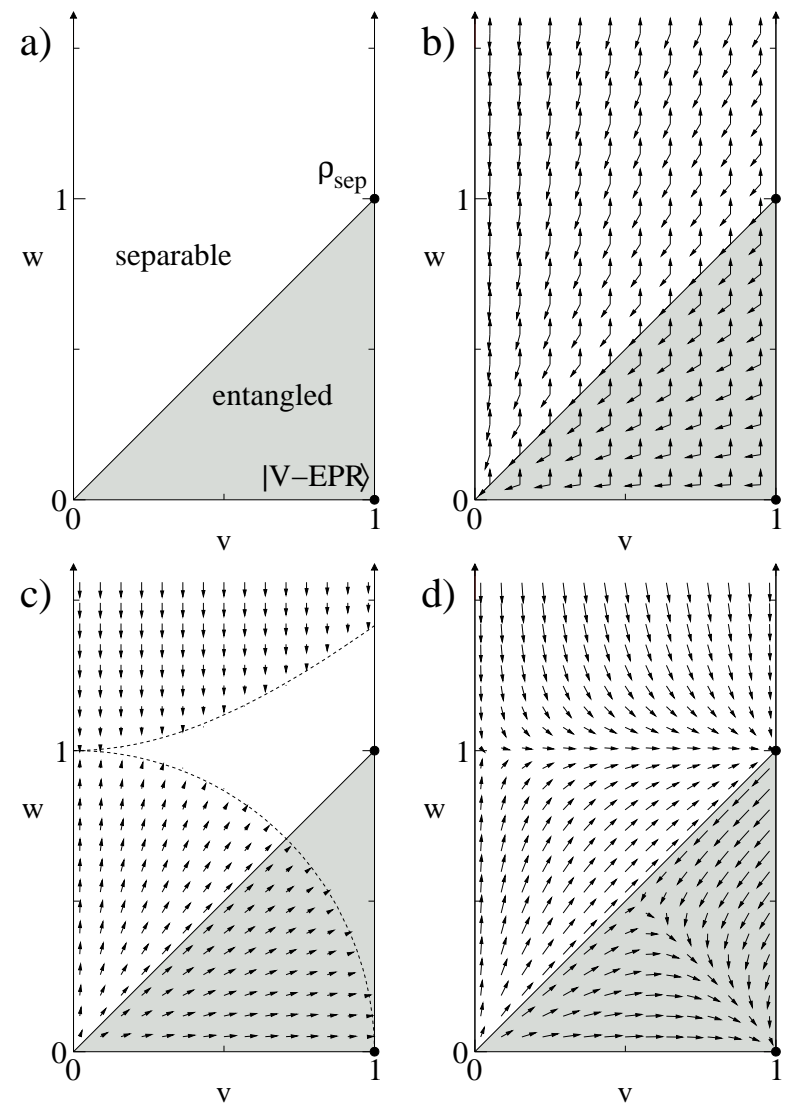

FIG. 5: a) Diagram characterizing mixed states according to their standard form. The entangled states are exactly those in the gray area. b) Transformations possible by one-copy operations: $w$ can be increased, or $w$ and $v$ can be decreased simultaneously. Thereby, the $|\mathrm{V}-\mathrm{EPR}\rangle$ can be transformed to any state, while $\rho_{\text {sep }}$ can generate any separable state. c) Additional transformations realizable by two-copy recurrence protocols. Thereby, it is not possible to reach $\rho_{\text {sep }}$ or $|\mathrm{V}-\mathrm{EPR}\rangle$. d) Three-copy protocols allow to distill all separable states towards $\rho_{\text {sep }}$ and all entangled ones towards $\mid \mathrm{V}$-EPR $\rangle$.

which restricts their degrees of freedom to one complex number each, so that it is easy to check that the best protocols are given by

$$
M_{A}=M_{B} \propto\left(\begin{array}{llll}
1 & 0 & 0 & 0 \\
0 & 1 & 1 & 0
\end{array}\right)
$$

and

$$
M_{A}^{\prime} \propto\left(\begin{array}{cccc}
1 & 0 & 0 & 0 \\
0 & 1 & 1 & 0
\end{array}\right), \quad M_{B}^{\prime} \propto\left(\begin{array}{llll}
0 & 1 & 1 & 0 \\
0 & 0 & 0 & 1
\end{array}\right) .
$$

The resulting transformations are $(v, w) \mapsto$ $\left(v, \sqrt{\frac{1+v^{2}+w^{2}}{2}}\right)$ and $(v, w) \mapsto \sqrt{\frac{2}{1+v^{2}+w^{2}}}(v, w)$, respectively. Fig. [5: shows where this gives an advantage over the one-copy protocol Fig. 5 b. Obviously, two-copy protocols do neither allow to distill separable state to $\rho_{\text {sep }}$ nor do they allow to distill entangled states to $|\mathrm{V}-\mathrm{EPR}\rangle$.
For three copies, though, the following two pairs of POVM operatos provide a way to distill all states:

$$
M_{A}=M_{B} \propto\left(\begin{array}{llllllll}
0 & 1 & 1 & 0 & 1 & 0 & 0 & 0 \\
0 & 0 & 0 & 1 & 0 & 1 & 1 & 0
\end{array}\right)
$$

distills all separable states to $\rho_{\text {sep }}$ by virtue of

$$
(v, w) \mapsto\left(v+\frac{v-v^{3}}{1+2 v^{2}+2 w^{2}}, \frac{w\left(2+2 v^{2}+w^{2}\right)}{1+2 v^{2}+2 w^{2}}\right)
$$

whereas

$$
\begin{aligned}
& M_{A}^{\prime} \propto\left(\begin{array}{cccccccc}
0 & 1 & 1 & 0 & -1 & 0 & 0 & 0 \\
0 & 0 & 0 & 1 & 0 & 1 & 1 & 0
\end{array}\right), \\
& M_{B}^{\prime} \propto\left(\begin{array}{cccccccc}
0 & 1 & 1 & 0 & 1 & 0 & 0 & 0 \\
0 & 0 & 0 & -1 & 0 & 1 & 1 & 0
\end{array}\right)
\end{aligned}
$$

distills entangled states towards a $|\mathrm{V}-\mathrm{EPR}\rangle$, and

$$
(v, w) \mapsto\left(\frac{v\left(6+3 v^{2}-2 w^{2}\right)}{3+6 v^{2}+6 w^{2}}, \frac{w\left(6-2 v^{2}+3 w^{2}\right)}{3+6 v^{2}+6 w^{2}}\right) .
$$

This is illustrated in Fig. $5 \mathrm{~b}$.

\section{SIV AS A RESOURCE}

\section{A. Introduction}

In its standard form, i.e., as a singlet, EoE can be used to teleport quantum bits and thus allows to overcome the restriction to LOCC. In this section, we will show that $\mathrm{SiV}$ is a resource in very much the same way, namely it allows to overcome the restrictions imposed by SSR in a bipartite setting. Despite the similarities, there are some major differences. Firstly, while for EoE there only exists one standard form (the maximally entangled state depending on the dimension of the system), for $\mathrm{SiV}$ there exist two different standard states: singlets $|0\rangle|N\rangle+|N\rangle|0\rangle$ with $\mathrm{SiV} N^{2}$ (as in Corollary 1) and the Gaussian distributed states with large variance (as in Theorem 2). Second, there are no pure states which carry only $\mathrm{SiV}-\mathrm{SiV}$ as a resource which is independent of $\mathrm{EoE}$ only exists for mixed states where resources are difficult to quantify. Finally, we will find that we need an infinite amount of nonlocality in order to completely overcome the restrictions imposed by the SSR - this is fundamentally different from EoE where one ebit of entanglement is sufficient to perfectly teleport one quantum bit.

In order to demonstrate (and partly quantify) that $\mathrm{SiV}$ is useful to overcome the restrictions imposed by the SSR, we will use the tasks of distinguishing and teleportation. It has been shown that with SSR there exists a perfect data hiding protocol [9] which allows to encode a classical bit in a bipartite state such that it cannot be revealed by LOCC [7]. This protocol can be extended to a protocol hiding $\log N$ bits in the Fourier states

$$
\left|\zeta_{k}^{N}\right\rangle=\frac{1}{\sqrt{N}} \sum_{n=0}^{N-1} e^{2 \pi i k n / N}|n\rangle_{A}|N-1-n\rangle_{B}
$$


These states can be distinguished perfectly by LOCC if no SSR are present (therefore, both parties measure in the Fourier basis and compare their outcomes), but with SSR, they become totally indistinguishable (see Theorem 5 below).

The second task we use to show that $\mathrm{SiV}$ is a resource is teleportation of a state with nonconstant local particle number: Alice holds one half of a state $|\phi\rangle_{A C}$ which she wants to teleport to Bob using an in general mixed helper state $\rho_{A B}$. Clearly, one ebit of EoE is necessary for this task, but if $V(|\phi\rangle)>0$, also $\mathrm{SiV}$ is needed [7]. We will show that one ebit of EoE is still sufficient, but the amount of SiV has to grow superlinearly with $V(|\phi\rangle)$ and is infinite for perfect teleportation.

\section{B. A general protocol}

Let us first quote a Theorem from [7] which will be very useful in the following.

Theorem $5([\mathbf{7}])$. In the presence of superselection rules, the states $\rho$ and $\mathcal{N}_{A \mid B}(\rho)$ cannot be distinguished by LOCC. Here, $\mathcal{N}_{A \mid B}$ is the "dephasing map"

$$
\mathcal{N}_{A \mid B}(\rho)=\sum_{n_{A}, n_{B}} P_{n_{A}}^{A} \otimes P_{n_{B}}^{B} \rho P_{n_{A}}^{A} \otimes P_{n_{B}}^{B},
$$

with $P_{n_{X}}^{X}$ the local projector onto the subspace with $n_{X}$ particles.

By this theorem, we can highly restrict the class of allowed protocols. Let us show this for the task of distinguishing, where Alice and Bob initially share $\rho=$ $\left|\zeta_{k}^{N}\right\rangle\left\langle\zeta_{k}^{N}\right| \otimes \sigma$ [cf. Eq. (19)], and they have to determine $k$. At the end of the protocol, Alice and Bob get an answer $k^{\prime}$ according to a probability distribution $\left\{p_{k^{\prime}}\right\}$. But if they had started with $\mathcal{N}_{A \mid B}(\rho)$ instead, Theorem [5 tells us that the probability distribution of their outcomes would have been just the same. Therefore, Alice and Bob can start their protocol by measuring their particle number operators $\hat{N}_{A}$ and $\hat{N}_{B}$-if they discard their outcomes, they just implemented $\mathcal{N}_{A \mid B}$, and their knowledge of $N_{A}$ and $N_{B}$ will not affect the average probability distribution which is solely relevant unless the figure of merit is nonlinear. The same holds for the teleportation scenario with respect to the partition $A \mid B C$, i.e., in this case only Alice is allowed to measure her particle number (this map is actually weaker than $\left.\mathcal{N}_{A \mid B C}\right)$. Note that for a pure state, measuring $\hat{N}_{A}$ also determines $N_{B C}$ (and thus implements $\mathcal{N}_{A \mid B C}$ ), which is different in the mixed state case and closely connected to the fact that mixed states alone are not sufficient for teleportation.

\section{Pure states}

Assume Alice wants to teleport her share of the state

$$
|\phi\rangle=\sum_{n} \alpha_{n}|n\rangle_{A}|-n\rangle_{C}
$$

to Bob using

$$
|\psi\rangle=\sum_{m} \beta_{m}|m\rangle_{A}|-m\rangle_{B}
$$

Here, we use a simplified notation, where $-\infty<n, m<$ $\infty, \sum\left|\alpha_{n}\right|^{2}=\sum\left|\beta_{m}\right|^{2}=1$, and the support of the $\alpha_{n}$, $\beta_{m}$ is bounded below such that the particle number can be made positive by adding ancillas. As shown before, Alice can start any protocol by measuring $N_{A}=K$, yielding

$$
\sqrt{p_{K}}\left|\chi_{K}\right\rangle_{A B C}=\sum_{n+m=K} \alpha_{n} \beta_{m}|n, m\rangle_{A}|-n\rangle_{C}|-m\rangle_{B}
$$

with included probability $p_{K}$. If Alice now measures in the Fourier basis and communicates her result, the originally tripartite state can be reconstructed by Bob and Charlie; this strategy is optimal as no information is lost and the state gets less nonlocal. Up to shifts in the particle number, the state is then

$$
\sqrt{p_{K}}\left|\chi_{K}\right\rangle_{B C}=\sum_{n} \alpha_{n} \beta_{K-n}|n\rangle_{B}|-n\rangle .
$$

We will use the average entanglement fidelity as the figure of merit,

$$
\bar{F}=\left\langle\sum_{K} p_{K}\left|\left\langle\phi \mid \chi_{K}\right\rangle\right|^{2}\right\rangle=\sum_{\Delta} \Pi(\Delta) C(\Delta)
$$

where $\Pi(\Delta)=\sum_{n}\left\langle p_{n} p_{n+\Delta}\right\rangle\left(p_{n}=\left|\alpha_{n}\right|^{2}\right)$ and $C(\Delta)=$ $\sum_{m} \beta_{m}^{*} \beta_{m-\Delta}$. The average $\langle\cdot\rangle$ is taken over all states $\phi$, where for teleportation we assume a unitarily invariant distribution. It is straightforward to check that local filtering operations cannot increase $\bar{F}$. Also, for the task of distinguishing it can be shown that $\bar{F}$ gives the optimal success probability for the inconclusive case [28]. For distinguishing, $\Pi_{D}(\Delta)=\max (N-|\Delta|, 0) / N^{2}$, while for teleportation, $\Pi_{T}(\Delta)=\left[\max (N-|\Delta|, 0)+\delta_{\Delta, 0}\right] / N(N+$ 1) 29]. In both cases, $\alpha_{n} \neq 0$ for $n=0, \ldots, N-1$.

We will analyze two natural types of helper states: states with constant distribution $\beta_{m}=1 / \sqrt{M}, m=$ $0, \ldots, M-1$, and states with Gaussian distribution with variance $V(\psi)$. One finds $C_{C}(\Delta)=\max (M-|\Delta|, 0) / M$ resp. $C_{G}(\Delta)=\exp \left[-\Delta^{2} / 2 V\right] \approx 1-\Delta^{2} / 2 V$. The resulting error probabilities for all four cases are given in Table I Note that for the Gaussian distributed $|\psi\rangle$, in both cases

$$
p_{\text {err }}=\frac{\langle V(\phi)\rangle}{4 V(\psi)}
$$

holds, i.e., the error probability is given by the ratio of the variances. (Actually, this even holds without taking the average over $\phi$.)

In all cases, the error vanishes only if the size of the helper state grows superlinearly with the size of the unknown state; thus, the scaling of $\mathrm{SiV}$ as a resource is 


\begin{tabular}{l||c|c}
\multirow{2}{*}{\multicolumn{1}{c||}{ helper }} & distinguish & teleport \\
\cline { 2 - 3 } constant & $p_{\text {err }}=\frac{(N+1)(N-1)}{3 M N}$ & $p_{\text {err }}=\frac{N}{3 M}$ \\
\hline Gaussian & $p_{\text {err }}=\frac{(N+1)(N-1)}{12 V(\psi)}$ & $p_{\text {err }}=\frac{N(N-1)}{12 V(\psi)}$
\end{tabular}

TABLE I: Error probabilities for distinguishing and teleportation where the helper state is either a maximally entangled state with Schmidt number $N$ or a Gaussian distributed state with variance $V(\psi)$.

unfavorable compared to the behaviour of EoE. This is a direct consequence of the direct sum structure in (3) which is opposed to the tensor product structure leading to EoE: while with a tensor product structure, $N$ particles generate a $2^{N}$-dimensional Hilbert space, for the direct sum structure the underlying space only has dimension $N+1$. This also holds for mixed states, where this is the size of the largest coherent subspace. For the same reason, the data hiding scheme Eq. (19) is optimal in the sense that the available Hilbert space has only dimension $N$.

\section{Mixed states}

Let us now demonstrate that separable mixed states with SiV can also be used as a shared reference frame 7]. First, we demonstrate how Alice and Bob can use the state $\rho_{\text {sep }}$ to distinguish the states $|01\rangle \pm|10\rangle$. By very much the same argument as before Alice and Bob can start their protocol by measuring their local particle number. By adding their outcomes, Alice and Bob immediately know whether they are dealing with the $|0\rangle|0\rangle /|1\rangle|1\rangle$ part of $\rho_{\text {sep }}$ or with the $\mid \mathrm{V}$-EPR $\rangle$. In the first case all information is lost while in the second case they can just proceed as if they had started with $\mid \mathrm{V}$-EPR $\rangle$ itself. This case occurs with probability $1 / 2$, i.e., all the $\mathrm{SiV}$ contained in $\rho_{\text {sep }}$ can be used. Clearly, this protocol can not be used for teleportation as $\hat{N}_{B C}$ cannot be implemented locally.

Let us now show that separable but nonlocal states can be used to overcome locality constraints arbitrarily well, i.e., they can serve as arbitrarily precise reference frames. Therefore, we use the separable state [7, 30]

$$
\rho_{\text {coh }}(\alpha)=\int \frac{\mathrm{d} \phi}{2 \pi}\left|\alpha e^{i \phi}\right\rangle\left\langle\alpha e^{i \phi}|\otimes| \alpha e^{i \phi}\right\rangle\left\langle\alpha e^{i \phi}\right|
$$

where for $\alpha>0$,

$$
\left|\alpha e^{i \phi}\right\rangle=e^{-\alpha^{2} / 2} \sum_{n=0}^{\infty} \frac{\alpha^{n}}{\sqrt{n !}} e^{i n \phi}
$$

is a coherent state with amplitude $\alpha e^{i \phi}$. It has been shown 7] that for $\alpha \rightarrow \infty, \rho_{\mathrm{coh}}(\alpha)$ can be used to distinguish $|01\rangle \pm|01\rangle$ with arbitrary precision. In the following, we will show that this state together with one $|\mathrm{E}-\mathrm{EPR}\rangle$ can be used to perfectly teleport a state with nonconstant local particle number and therefore may serve as an arbitrarily precise reference frame.

First, let us use Theorem 4 to show that this state has indeed infinite $\mathrm{SiV}$ for $\alpha \rightarrow \infty$. Therefore, it is enough to note that

$$
\begin{array}{r}
\rho_{\mathrm{coh}}(\alpha)=\sum_{N=0}^{\infty} p_{N}\left|\theta_{N}\right\rangle\left\langle\theta_{N}\right| ; \quad p_{N}=e^{-2 \alpha^{2}} \frac{\left(2 \alpha^{2}\right)^{N}}{N !} \\
\left|\theta_{N}\right\rangle=\frac{1}{\sqrt{2^{N}}} \sum_{n=0}^{N}\left(\begin{array}{c}
N \\
n
\end{array}\right)^{1 / 2}|n, N-n\rangle,
\end{array}
$$

and thus $V_{F}^{\mathrm{SSR}}\left(\rho_{\mathrm{coh}}(\alpha)\right)=V_{c}^{\mathrm{SSR}}\left(\rho_{\mathrm{coh}}(\alpha)\right)=\alpha^{2} / 2 \rightarrow \infty$ for $\alpha \rightarrow \infty$. In is interesting to note that each of the $\left|\theta_{N}\right\rangle$ approximates a state with Gaussian distribution such that $\rho_{\text {coh }}(\alpha)$ might be considered as the EoE-free mixed state version of Gaussian distributed states.

In order to see how a mixed state can be used to teleport, let Alice and Charlie initially share $|\phi\rangle=$ $\alpha|01\rangle+\beta|10\rangle$ (the proof is completely analogous for qu$d$-its), and assume Alice wants to teleport her share to Bob. Therefore, Alice and Bob are provided with an $|\mathrm{E}-\mathrm{EPR}\rangle_{A B}$ and with some mixed state

$$
\rho=\sum_{\substack{n, m, n^{\prime}, m^{\prime} \\ n+m=n^{\prime}+m^{\prime}}} \rho_{n, m}^{n^{\prime}, m^{\prime}}|n\rangle_{A}\left\langle n^{\prime}|\otimes| m\right\rangle_{B}\left\langle m^{\prime}\right|
$$

where the condition $n+m=n^{\prime}+m^{\prime}$ comes from the SSR, Eq. (11). For simplicity, let us assume that all $\rho_{n, m}^{n^{\prime}, m^{\prime}}$ are nonnegative. Alice once more starts by measuring her local particle number operator on $|\phi\rangle\langle\phi| \otimes \rho$. (In this step, we do not have to care about the $|\mathrm{E}-\mathrm{EPR}\rangle$ which has constant local particle number.) For a measurement outcome $n$, the resulting state (probability included) is

$$
\begin{aligned}
\sum_{m} & {\left[|\alpha|^{2} \rho_{n, m-1}^{n, m-1}|0, n\rangle_{A}\langle 0, n|\otimes| m-1\rangle_{B}\langle m-1|\otimes| 1\rangle_{C}\langle 1|\right.} \\
& +|\beta|^{2} \rho_{n-1, m}^{n-1, m}|1, n-1\rangle_{A}\langle 1, n-1|\otimes| m\rangle_{B}\langle m|\otimes| 0\rangle_{C}\langle 0| \\
& +\alpha \beta^{*} \rho_{n, m-1}^{n-1, m}|0, n\rangle_{A}\langle 1, n-1|\otimes| m-1\rangle_{B}\langle m|\otimes| 1\rangle_{C}\langle 0| \\
& \left.+\alpha^{*} \beta \rho_{n-1, m}^{n, m-1}|1, n-1\rangle_{A}\langle 0, n|\otimes| m\rangle_{B}\langle m-1|\otimes| 0\rangle_{C}\langle 1|\right]
\end{aligned}
$$

As Alice's share now has constant particle number and lies within a two-dimensional subspace, she can use the $|\mathrm{E}-\mathrm{EPR}\rangle_{A B}$ to teleport her share to Bob. If we label the two teleported basis states $|\hat{a}\rangle=|0, n\rangle,|\hat{b}\rangle=|1, n-1\rangle$, Bob and Charlie then share the state

$$
\begin{aligned}
\sum_{m} & {\left[|\alpha|^{2} \rho_{n, m-1}^{n, m-1}|\hat{a}, m-1\rangle_{B}\langle\hat{a}, m-1|\otimes| 1\rangle_{C}\langle 1|\right.} \\
& +|\beta|^{2} \rho_{n-1, m}^{n-1, m}|\hat{b}, m\rangle_{B}\langle\hat{b}, m|\otimes| 0\rangle_{C}\langle 0| \\
& +\alpha \beta^{*} \rho_{n, m-1}^{n-1, m}|\hat{a}, m-1\rangle_{B}\langle\hat{b}, m|\otimes| 1\rangle_{C}\langle 0| \\
& \left.+\alpha^{*} \beta \rho_{n-1, m}^{n, m-1}|\hat{b}, m\rangle_{B}\langle\hat{a}, m-1|\otimes| 0\rangle_{C}\langle 1|\right]
\end{aligned}
$$


Bob now projects onto the subspaces spanned by the pairs of states $\left|0_{m}\right\rangle \equiv|\hat{a}, m-1\rangle$ and $\left|1_{m}\right\rangle \equiv|\hat{b}, m\rangle$ and obtains

$$
\begin{array}{r}
\quad|\alpha|^{2} \rho_{n, m-1}^{n, m-1}|0\rangle_{B}\langle 0|\otimes| 1\rangle_{C}\langle 1| \\
+|\beta|^{2} \rho_{n-1, m}^{n-1, m}|1\rangle_{B}\langle 1|\otimes| 0\rangle_{C}\langle 0| \\
+\quad \alpha \beta^{*} \rho_{n, m-1}^{n-1, m}|0\rangle_{B}\langle 1|\otimes| 1\rangle_{C}\langle 0| \\
+\quad \alpha^{*} \beta \rho_{n-1, m}^{n, m-1}|1\rangle_{B}\langle 0|\otimes| 0\rangle_{C}\langle 1|
\end{array}
$$

(where we omitted the subscript $m$ ). By looking at the average fidelity with the original state, we find that the error vanishes iff

$$
\sum_{n, m} \rho_{n, m-1}^{n, m-1}=\sum_{n, m} \rho_{n-1, m}^{n-1, m}=\sum_{n, m} \rho_{n-1, m}^{n, m-1} .
$$

Since $\rho$ is positive this implies that $\rho_{n, m-1}^{n, m-1} \approx \rho_{n-1, m}^{n-1, m} \approx$ $\rho_{n-1, m}^{n, m-1}$ for most $n, m$, as one would expect from Eq. (22). It is straightforward to check that Eq. (23) holds for $\rho_{\text {coh }}(\alpha)$ for $\alpha \rightarrow \infty$, and that the $2 \times 2$ subblocks of the density matrix really approximate pure states.

One might expect that $N \rightarrow \infty$ copies of $\rho_{\text {sep }}$ could be used just the same way, but the situation is quite different: filtering operations which bring $\rho_{\text {sep }}^{\otimes N}$ into a form (21) destroy the off-diagonal elements of the density matrix with high probability so that (23) cannot be satisfied; therefore it is questionable whether multiple copies of $\rho_{\text {sep }}$ can be used as an arbitrarily precise reference frame. On the other hand, this is not so much different from the pure state scenario: while multiple copies of a $\mid \mathrm{V}$-EPR $\rangle$ might indeed be used as a perfect reference frame, these states carry an amount of entanglement which grows linearly with the precision of the reference frame, whereas a single Gaussian distributed state with large SiV only has logarithmic - and thus in some sense vanishing - entanglement and is therefore much closer to the case of separable reference frames.

Let us note that the teleportation scenario can be altered by joining $B$ and $C$. This is no longer teleportation, of course, and can be accomplished by LOCC without SSR. On the other hand, it is still an impossible task if SSR are present and is thus suitable to characterize mixed states as reference frames without the need for additional entanglement.

\section{E. Hiding quantum states}

Let us close by showing that the data hiding protocol given in 7] resp. its extension Eq. (19) can be used to construct a mixed state scheme to hide quantum data as well. At a first glance, one might try to encode the two degrees of freedom of a qubit in the phases of the state $|02\rangle+e^{i \phi}|11\rangle+e^{i \phi^{\prime}}|20\rangle$, but this cannot be accomplished by a linear map. Therefore, we encode the qubit $\alpha|01\rangle+\beta|10\rangle$ in one of the states $\left|\phi_{0}\right\rangle=\alpha|01\rangle+\beta|10\rangle$, $\left|\phi_{1}\right\rangle=\beta|01\rangle+\alpha|10\rangle$ with equal probabilities which is then distributed between Alice and Bob. Additionally, Alice and Bob are provided with a state $\left|\psi_{0 / 1}\right\rangle=|02\rangle \pm|20\rangle$ which encodes the state Alice and Bob actually share. Thus, Alice and Bob share a state which they cannot distinguish from the totally mixed state by LOCC (Theorem [1), but they can perfectly recover the original qubit if they join. This scheme can be extended to hide $N$-level quantum states using one of the states

$\left|\phi_{k}\right\rangle=\sum_{n=0}^{N-1} \alpha_{n+k \bmod N}|n, N-1-n\rangle ; \quad k=0, \ldots, N-1$.

Together with the state encoding $k, N^{2}-1$ particles are needed, and the associated Hilbert space dimension is $N^{2}$.

\section{CONCLUSIONS}

Adding restrictions to the operations permissible on a quantum system gives rise to a new resource which in turn allows to overcome this restriction. The restriction to LOCC, for example, leads to EoE as a nonlocal resource. Adding SSR to a bipartite system leads to an additional resource, the superselection induced variance $\mathrm{SiV}$. We could show that $\mathrm{SiV}$ and EoE together completely characterize bipartite states in the asymptotic limit. Thereby, two different kind of standard forms arise, namely singlets and Gaussian distributed states with logarithmic EoE.

The search for states which only carry SiV led us to mixed states, where we considered entanglement and variance of formation. We could show that the concept of entanglement does not have to be changed and thus there exist states which carry $\mathrm{SiV}$ but no EoE, and we provided explicit formulas for the case of qubits. As to distillation, we could show that both EoE and SiV can be distilled, and we provided various ways to do that. Thereby, we found that there exist mixed standard states for $\mathrm{SiV}$ which do not carry EoE. While it is possible to extend recurrence protocols such that they work with SSR by using a third copy as a reference frame, it is unlikely that protocols with asymptotic yield work.

Finally, we showed that $\mathrm{SiV}$ is a resource which allows to overcome the restrictions imposed by the SSR, but we also saw that there are fundamental differences to EoE as the size of the reference frame has to grow superlinearly with the problem size, which is due to direct sum structure of the underlying Hilbert space.

\section{Acknowledgments}

We acknowledge helpful discussions with K. G. Vollbrecht and M. M. Wolf. This work was supported in part by the E.C. (RESQ QUIPRODIS) and the Kompetenznetzwerk "Quanteninformationsverarbeitung" der Bayerischen Staatsregierung. 


\section{APPENDIX: PROOF OF EQ. (15)}

In order to show Eq. [15), we need the inequality $S\left(p_{i} \rho_{i}\right) \leq p_{i} S\left(\rho_{i}\right)+H\left(p_{i}\right)$ (see, e.g., 21] for a proof). Furthermore, note that $P_{n}=\sum_{i=0}^{n} P_{i}^{A} \otimes P_{n-i}^{B}$. Toghether, this gives the estimate

$$
\begin{aligned}
E\left(\frac{P_{n}|\psi\rangle}{\sqrt{\left\langle\psi\left|P_{n}\right| \psi\right\rangle}}\right) & =S\left(\frac{\operatorname{tr}_{B} \sum_{i=0}^{n} P_{i}^{A} \otimes P_{n-i}^{B}|\psi\rangle\langle\psi| P_{i}^{A} \otimes P_{n-i}^{B}}{\left\langle\psi\left|P_{n}\right| \psi\right\rangle}\right) \\
& \leq \sum_{i=0}^{n} \frac{\left\langle\psi\left|P_{i}^{A} \otimes P_{n-i}^{B}\right| \psi\right\rangle}{\left\langle\psi\left|P_{n}\right| \psi\right\rangle} S\left(\frac{\operatorname{tr}_{B} P_{i}^{A} \otimes P_{n-i}^{B}|\psi\rangle\langle\psi| P_{i}^{A} \otimes P_{n-i}^{B}}{\left\langle\psi\left|P_{i}^{A} \otimes P_{n-i}^{B}\right| \psi\right\rangle}\right)+H\left(\left\{\frac{\left\langle\psi\left|P_{i}^{A} \otimes P_{n-i}^{B}\right| \psi\right\rangle}{\left\langle\psi\left|P_{n}\right| \psi\right\rangle}\right\}_{i=0}^{N}\right) .
\end{aligned}
$$

Clearly, the Shannon entropy $H$ is bounded by $\log (n+1) \leq \log (N+1)$, and thus the 1.h.s. of Eq. (15), i.e., the entanglement averaged over $n$, is bounded by

$$
\sum_{n=0}^{N} \sum_{i=0}^{n}\left\langle\psi\left|P_{i}^{A} \otimes P_{n-i}^{B}\right| \psi\right\rangle S\left(\frac{\operatorname{tr}_{B} P_{i}^{A} \otimes P_{n-i}^{B}|\psi\rangle\langle\psi| P_{i}^{A} \otimes P_{n-i}^{B}}{\left\langle\psi\left|P_{i}^{A} \otimes P_{n-i}^{B}\right| \psi\right\rangle}\right)+\log [N+1] .
$$

The sum can be extended to $i=0, \ldots, N, n-i=0, \ldots, N$ as $|\psi\rangle$ has at most $N$ particles, and by the convexity of the von Neumann entropy, Eq. (15) follows.

[1] C. H. Bennett, H. J. Bernstein, S. Popescu, and B. Schumacher, Phys. Rev. A 53, 2046 (1996), quant$\mathrm{ph} / 9511030$.

[2] C. H. Bennett, G. Brassard, C. Crépeau, R. Jozsa, A. Peres, and W. K. Wootters, Phys. Rev. Lett. 70, 1895 (1993).

[3] C. H. Bennett and S. J. Wiesner, Phys. Rev. Lett. 69, 2881 (1992).

[4] P. Zanardi, Phys. Rev. Lett. 87, 077901 (2001), quant-ph/0103030 P. Zanardi, D. Lidar, and S. Lloyd, Phys. Rev. Lett. 92, 060402 (2004), quant-ph/0308043

[5] H. Barnum, E. Knill, G. Ortiz, and L. Viola, Phys. Rev. A 68, 032308 (2003), quant-ph/0207149 H. Barnum, E. Knill, G. Ortiz, R. Somma, and L. Viola, Phys. Rev. Lett. 92, 107902 (2004), quant-ph/0305023

[6] S. Popescu, personal communication.

[7] F. Verstraete and J. I. Cirac, Phys. Rev. Lett. 91, 010404 (2003), quant-ph/0302039.

[8] H. M. Wiseman and J. A. Vaccaro, Phys. Rev. Lett. 91, 097902 (2003), quant-ph/0210002 S. D. Bartlett and H. M. Wiseman, Phys. Rev. Lett. 91, 097903 (2003), quant-ph/0303140 H. M. Wiseman, S. D. Bartlett, and J. A. Vaccaro, quant-ph/0309046 J. A. Vaccaro, F. Anselmi, and H. M. Wiseman, Int. J. Quant. Inf., 1, 427 (2003), quant-ph/0311028

[9] B. M. Terhal, D. P. DiVincenzo, and D. W. Leung, Phys. Rev. Lett. 86, 5807 (2001).

[10] A. Kitaev, D. Mayers, and J. Preskill (2003), quant$\mathrm{ph} / 0310088$.

[11] S. D. Bartlett, T. Rudolph, and R. W. Spekkens (2004), quant-ph/0403161.

[12] N. Schuch, F. Verstraete, and J. Cirac, Phys. Rev. Lett. 92, 087904 (2004), quant-ph/0310124.

[13] M. Nielsen, Phys. Rev. Lett. 83, 436 (1999), quant$\mathrm{ph} / 9811053$.

[14] C. H. Bennett, D. P. DiVincenzo, J. A. Smolin, and W. K. Wootters, Phys. Rev. A 54, 3824 (1996), quant- ph/9604024.

[15] C. H. Bennett, G. Brassard, S. Popescu, B. Schumacher, J. A. Smolin, and W. K. Wootters, Phys. Rev. Lett. 76, 722 (1996), quant-ph/9511027.

[16] D. Jonathan and M. B. Plenio, Phys. Rev. Lett. 83, 1455 (1999), quant-ph/9903054.

[17] If one includes ancillas, the Theorem only holds up to shifts in the local particle number performed on the output states, i.e., Eq. (5) has to be replaced by $\exists \nu_{i} \in \mathbb{N}$ $\forall n=0, \ldots, N: \boldsymbol{\lambda}^{n} \prec \sum_{i} p_{i} \boldsymbol{\mu}_{i}^{n+\nu_{i}}$. Formally, up to local unitaries each state is then described by an equivalence class of SSR-ordered Schmidt vectors which are equal up to a shift in the particle number, and there have to exist vectors in these equivalence classes which satisfy Eq. (5). The proof transfers directly if one replaces $\hat{\psi}^{n}$ by $\hat{\psi}^{n+\nu_{i}}$.

[18] H.-K. Lo and S. Popescu, Phys. Rev. A 63, 022301 (2001), quant-ph/9707038.

[19] G. Vidal, J. Mod. Opt. 47, 355 (2000), quantph/9807077.

[20] T. M. Cover and J. A. Thomas, Elements of Information Theory (Wiley-Interscience, 1991).

[21] M. A. Nielsen and I. A. Chuang, Quantum Computation and Quantum Information (Cambridge University Press, 2000).

[22] P. M. Hayden, M. Horodecki, and B. M. Terhal, J. Phys. A 34, 6891 (2001), quant-ph/0008134.

[23] E.g, for class of $2 \times 2$ qubit states given by $|\phi\rangle=$ $\alpha|00\rangle_{A}|11\rangle_{B}+\beta|01\rangle_{A}|10\rangle_{B}+\beta|10\rangle_{A}|01\rangle_{B}+\alpha|11\rangle_{A}|00\rangle_{B}$ with $\alpha=\sqrt{p / 2}, \beta=\sqrt{(1-p) / 2}$, and $p<1 / 2, V_{F}^{\text {SSR }}$ is not superadditive with respect to the two subsystems, since $V(|\phi\rangle)=4 p$ and $V_{F}^{\mathrm{SSR}}\left(\operatorname{tr}_{i}[|\phi\rangle\langle\phi|]\right)=4 p(1-p)$.

[24] A. Pomeransky, Phys. Rev. A 68, 032317 (2003), quant$\mathrm{ph} / 0305056$.

[25] W. K. Wootters, Phys. Rev. Lett. 80, 2245 (1998), quant$\mathrm{ph} / 9709029$.

[26] F. Verstraete, J. Dehaene, and B. DeMoor, Phys. Rev. A 
64, 010101(R) (2001), quant-ph/0011111.

[27] Some special cases for $\rho$ have to be considered separately. Note that if $v=0 \rho$ can be created by LOCC, and $v=0$ follows if $w_{01}=0$ or $w_{10}=0$. If $w_{00}=w_{11}=0$, filtering still works in both directions if $w_{00}$ and $w_{11}$ are deleted from the filtering operators. In case $w_{00}=0$, $w_{11}>0$ or vice versa, the situation gets more complicated. In order to distill $\rho$, one adds $|00\rangle\langle 00|$ with some weight which does not destroy the entanglement; then, $\rho$ can be distilled to $|\mathrm{V}-\mathrm{EPR}\rangle$, from where it can be easily reconstructed.

[28] A. Chefles, Contemporary Physics 41, 401 (2000), quant$\mathrm{ph} / 0010114$.

[29] K. Banaszek, Phys. Rev. A 62, 024301 (2000), quant$\mathrm{ph} / 0002088$.

[30] T. Rudolph and B. C. Sanders, Phys. Rev. Lett. 87, 077903 (2001), quant-ph/0103147. 